\title{
Recent Developments on Kaon Condensation and Its Astrophysical Implications
}

\author{
Gerald E. Brown, ${ }^{a}$ Chang-Hwan Lee, ${ }^{b}$ Mannque Rho ${ }^{c}$ \\ ${ }^{a}$ Department of Physics and Astronomy, State University of New York, Stony \\ Brook, NY 11794, USA \\ ${ }^{\mathrm{b}}$ Department of Physics, Pusan National University, Busan 609-735, Korea \\ ' Institut de Physique Théorique, CEA Saclay, 91191 Gif-sur-Yvette cédex, France
}

\begin{abstract}
We discuss three different ways to arrive at kaon condensation at $n_{c} \simeq 3 n_{0}$ where $n_{0}$ is nuclear matter density: (1) Fluctuating around the $n=0$ vacuum in chiral perturbation theory, (2) fluctuating around $n_{V M}$ near the chiral restoration density $n_{\chi}$ where the vector manifestation of hidden local symmetry is reached and (3) fluctuating around the Fermi liquid fixed point at $\sim n_{0}$. They all share one common theoretical basis, "hidden local symmetry." We argue that when the critical density $n_{c}<n_{\chi}$ is reached in a neutron star, the electrons turn into $K^{-}$mesons, which go into an S-wave Bose condensate. This reduces the pressure substantially and the neutron star goes into a black hole. Next we develop the argument that the collapse of a neutron star into a black hole takes place for a star of $M \simeq 1.5 M_{\odot}$. This means that Supernova 1987A had a black hole as result. We also show that two neutron stars in a binary have to be within $4 \%$ of each other in mass, for neutron stars sufficiently massive that they escape helium shell burning. For those that are so light that they do have helium shell burning, after a small correction for this they must be within $4 \%$ of each other in mass. Observations support the proximity in mass inside of a neutron star binary. The result of strangeness condensation is that there are $\sim 5$ times more low-mass black-hole, neutron-star binaries than double neutron-star binaries although the former are difficult to observe.
\end{abstract}




\section{Contents}

1 INTRODUCTION 3

2 KAON CONDENSATION

2.1 Kaon Condensation As Restoration Of Explicit Chiral Symmetry Breaking

5

2.2 Strangeness Condensation By Expanding About the Fixed Point Of the Harada-Yamawaki Vector Manifestation

2.3 Hadronic Freedom, the Flash Point And Why the Constituent Quark Model Is Relevant

12

2.4 Calculation Of the Critical Density $n_{c}$ For Kaon Condensation 15

2.5 Kaon Condensation From the Fermi-Liquid Fixed Point 17

2.6 Delayed Collapse Of Neutron Star 18

3 A SCENARIO FOR A LARGE NUMBER OF LOW-MASS BLACK HOLES IN THE GALAXY 19

3.1 Hypercritical Accretion Onto First-Born Neutron Star During Common Envelope Evolution

20

3.2 Formation Of Double Neutron Star Binaries 24

3.3 Neutron Star - White Dwarf Binaries 27

3.4 Are There Two Branches of Neutron Stars? 28

3.5 New Measurement Of the Neutron Star Mass in J0751+1807 29

3.6 Neutron Stars In Globular Clusters

3.7 Convective Carbon Burning And Compact Star Masses 31

3.8 Accretion Onto Neutron Star After Supernova Explosion 33

3.9 Observability Premium For Unseen Black Hole - Neutron Star Binaries 34

4 CONCLUSIONS

References 38 


\section{INTRODUCTION}

While the phase structure of hadronic matter at high temperature both below and above the chiral phase transition temperature $T_{\chi}$ is being mapped out by laboratory experiments with invaluable help from lattice QCD, the situation with high density is vastly different. There is little information about matter above the nuclear matter density $n_{0} \approx 0.16 \mathrm{fm}^{-3}$ from laboratory experiments and there is practically no guidance from QCD proper since lattice - the only non-perturbative QCD tool available - cannot handle high density and the reliable perturbative QCD approach can access density regime only at asymptotic density where such novel phenomena like color-flavor-locked superconductivity can take place. To theorists' disappointments, though, this density regime may be totally irrelevant to nature. We have a complicated landscape of phases theoretically predicted near and above a chiral restoration $n_{\chi}$ which is as rich as the phase structure of water but they are all based on models, the reliability of which is uncertain given the paucity of experimental supports and lack of reliable theoretical control. Consequently the plethora of different scenarios for dense stellar systems such as neutron stars, black holes etc. available in the literature offer little guidance for understanding compact star physics.

The aim of this paper is to exploit a systematic effective field theory framework from three different vantage points to describe with some confidence the phase structure of dense matter crucially relevant to the formation of stable compact stars and its implications on the population of neutron stars and light-mass black holes. These approaches, particularly the second and the third, rely on one common strategy that hadronic systems under extreme conditions can be accessed reliably by hidden local symmetry [1] that incorporates the scaling property of chiral symmetry [2]. Our reasoning will be backed by detailed analysis of astrophysical observations.

This paper consists of two parts, one on an important phase change in hadronic physics and the other on collapse to black holes in astrophysics. Our aim is to bridge these seemingly disparate branches of physical phenomena. In the first part, we discuss recent developments on the most likely phase transition in hadronic matter, namely, kaon condensation, as density increases beyond the nuclear matter density to $\sim 3 n_{0}$. We will develop the thesis that this is the first - and perhaps the last - crucial phase change at high density that matters for the fate of compact stars, leaving wide-open the possibility of other forms of higher-density phases involving quark matter etc. In the second part, we give compelling arguments why astrophysical observations strongly support that neutron stars of mass greater than $\sim 1.5 M_{\odot}$ (where $M_{\odot}$ is the solar mass) cannot be stable, as a consequence of which any compact star more massive than the maximum stable mass must be in the form of a black 
hole. These two developments will then be joined to arrive at the conclusion that kaon condensation at $\sim 3 n_{0}$ implies $\sim 5$ times more low-mass black-hole, neutron-star binaries than double neutron-star binaries.

\section{KAON CONDENSATION}

Although QCD cannot provide at present useful and quantitative information for hadronic interactions at high density relevant to the physics of compact stars, there are three vantage points at which we have available reliable effective field theory tools to work with. The first is the matter-free $T=n=0$ vacuum about which fluctuations can be described by effective chiral field theory. Here there is a wealth of experimental data to guide model building and extrapolating beyond the normal matter density. The second is the other extreme regime of high temperature and/or density at which chiral symmetry is supposed to be restored, namely, the "vector manifestation fixed point" in hidden local symmetry formulated by Harada and Yamawaki [1] characterized by the gauge coupling going to zero and the interactions between hadrons becoming weak. The third is in between the two limits, i.e., in the vicinity of nuclear matter density at which the Fermi-liquid fixed point is located. Here both theoretical and experimental information accumulated since many decades in nuclear physics offers both guidance and control near the nuclear matter density and indicates what needs to be taken into account beyond. The results of these three approaches giving the critical density $n_{c} \lesssim 3 n_{0}$ have been reported before. What is new is the conceptual link between them provided by the recent development of chiral dynamics in hidden local symmetry theory recently supported by a holographic dual approach in string theory that gives an effective field theory of QCD in terms of (infinite towers of both) vector mesons and baryons and pions.

We will approach the state of matter believed to be present in the interior of compact stars from these three vantage points and converge to the result that kaons must condense in the vicinity of $3 n_{0}$. This then leads us to propose that kaon condensation is the most likely phase transition to take place in hadronic matter in the density regime that is relevant to the fate of compact stars. Any other possible phases such as e.g., quark matter, color superconductivity etc. that can appear at higher densities will then be academic issues for stellar objects, although interesting from a purely theoretical point of view. As we shall outline, the metastable behavior of SN1987A which gave off neutrinos for $\sim 12$ seconds and then disappeared in all respects is symptomatic of a condensate of negative charge kaons. In a number of papers we have outlined how evidence of a condensate which sends neutron stars into low-mass black holes, black holes not much more massive than neutron stars, is already present, and to us quite convincing, in the spectrum of masses in neutron-star binaries. 
It seems highly likely that the densities $\sim 3 n_{0}$ are reached in neutron stars. It is touch or go whether they were reached in the progenitor of SN1987A. However, they certainly are reached in the evolution of binary neutron stars in the case where the two progenitor giant masses are more than $4 \%$ different from each other in which case the first born neutron star finds itself in the red giant envelope of the companion giant as the latter evolves. This neutron star accretes matter from the evolving companion giant hypercritically until its mass is increased $\sim 0.7 M_{\odot}$, reaching densities enough for kaon condensation which sends it into a low-mass black hole.

If, on the other hand, the two progenitor giants are within $4 \%$ of each other in mass, they will burn helium at the same time and avoid the red giant stage of the second giant, which will then end up as a neutron star.

\subsection{Kaon Condensation As Restoration Of Explicit Chiral Symmetry Break- ing}

As the first estimate of the critical density for kaon condensation, we approach dense hadronic matter from the $T=n=0$ vacuum. This can be efficiently done by resorting to Weinberg's "folk theorem" [3] using effective chiral field theory. The basic idea is to construct an EFT Lagrangian that takes into account all relevant degrees of freedom at low energy and all pertinent symmetry constraints, in particular, chiral symmetry and do a systematic chiral perturbation theory calculation to as high an order as feasible. This procedure is by now fairly well established for pion-nucleon interactions as well as, to some degree, for finite nuclei and is being currently extended to nuclear matter [4]. The first approach made in this spirit is the work by Kaplan and Nelson [5]. In this subsection, we describe a toy description that while perhaps oversimplified, nonetheless brings out the essential feature of the physics involved while ignoring what we consider to be inessential complications. The key idea here is that kaon condensation can be viewed as the restoration by baryon density of chiral symmetry explicitly broken by the strange quark mass.

To formulate this idea in the most economical way, it was found to be simplest to take the $\mathrm{V}$-spin projection of the Goldstone boson sphere onto the $\mathrm{V}$-spin circle, composed of $K^{-}$and $\sigma$ in the $S U(3)$ chiral Lagrangian [6]. The Hamiltonian for explicit $K^{-}$chiral symmetry breaking brings in the kaon contribution to the explicit chiral symmetry breaking in the nucleon $\Sigma_{K N}$ and in the kaon mass $m_{K}$. The Hamiltonian for explicit chiral symmetry breaking which is of $O\left(p^{2}\right)$ in the chiral power counting - is

$$
H_{\chi S B}=\Sigma_{K N}\langle\bar{N} N\rangle \cos \theta+\frac{1}{2} m_{K}^{2} f_{\pi}^{2} \sin ^{2} \theta
$$




$$
\simeq \Sigma_{K N}\langle\bar{N} N\rangle\left(1-\frac{\theta^{2}}{2}\right)+\frac{1}{2} m_{K}^{2} f_{\pi}^{2} \theta^{2}
$$

where the last expression is obtained for small fluctuations $\theta$. Here we have taken mean field in the baryon sector. Dropping the term independent of $\theta$, we find

$$
m_{K}^{\star}{ }^{2}=m_{K}^{2}\left(1-\frac{\Sigma_{K N}\langle\bar{N} N\rangle}{f_{\pi}^{2} m_{K}^{2}}\right)^{1 / 2} .
$$

The $\Sigma_{K N}$ is

$$
\Sigma_{K N}=\frac{\left(m_{u}+m_{s}\right)\langle N|\bar{u} u+\bar{s} s| N\rangle}{\left(m_{u}+m_{d}\right)\langle N|\bar{u} u+\bar{d} d| N\rangle} \sigma_{\pi N}
$$

where $m_{u, d, s}$ are the current quark masses and $\sigma_{\pi N}$ is the pion $\sigma$-term. Lattice calculations [7] give

$$
\Sigma_{K N} \simeq 389(14) \mathrm{MeV}
$$

We can go one order higher in the "effective mass" of the kaon (2) by incorporating what is called "range term" which turns out be important quantitatively [8]. It amounts to changing $\Sigma_{K N}$ to

$$
\Sigma_{K N}^{\mathrm{eff}}=\left(1-0.37 \frac{\omega_{K^{-}}^{2}}{m_{K}^{2}}\right) \Sigma_{K N}
$$

where $\omega_{K^{-}}$is the (anti)kaon energy

$$
\omega_{K^{-}}=V_{T W}^{K^{-}}+\sqrt{k^{2}+{m_{K}^{-}}^{2}}
$$

In addition to the scalar attraction (which can be thought of as giving the kaon an effective mass), there is the vector attraction given by the TomozawaWeinberg term [9] - which is the leading term, i.e., $O(p)$, in the chiral counting

$$
V_{\mathrm{TW}}^{K^{-}}=-\frac{3}{8 f_{\pi}^{2}} n \approx-60 \mathrm{MeV} \frac{n}{n_{0}}
$$

where $n_{0}$ is nuclear matter density and we have taken the empirical value for the pion decay constant 


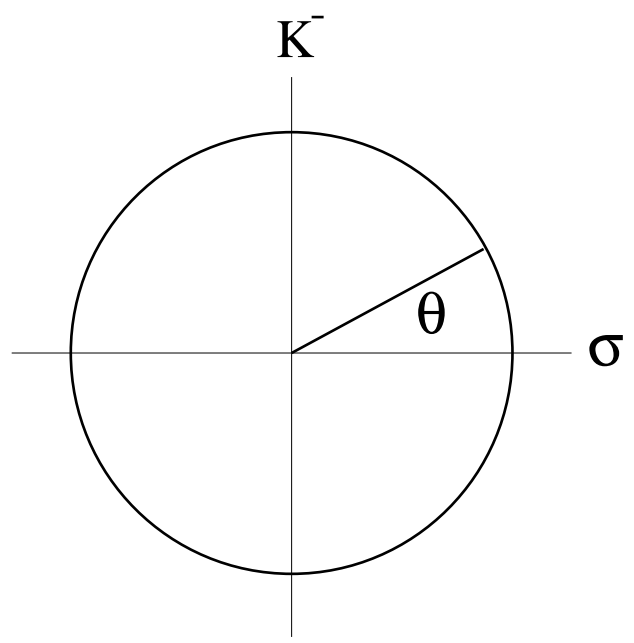

Fig. 1. Projection onto the $\sigma-K^{-}$plane. The angular variable $\theta$ represents fluctuation toward kaon mean field.

$$
f_{\pi} \simeq 93 \mathrm{MeV} .
$$

In a neutron star the electron chemical potential $\mu_{e^{-}}$, which in the progenitor of SN1987A was $\sim 220 \mathrm{MeV}[10]$ is the driving force towards kaon condensation [11]. Once the in-medium kaon mass $m_{K^{-}}^{\star}$ is less than $\mu_{e}$ then the degenerate electrons in the electron cloud in the neutron star can lower the energy by changing into a kaon condensate. The kaons are bosons, so they will immediately go into an S-state condensate, which lowers the pressure substantially from that of the degenerate electrons in the cloud in the neutron star. After this drop in pressure, the neutron star will go into a black hole in a light-crossing time.

The above captures the calculation of kaon condensation as it stood for many years that indicated that kaons could condense at $n_{c} \lesssim 3 n_{0}$. The principal lesson we learn here is that baryon density plays a prominent role, namely, that the underlying mechanism for condensation is the restoration of the explicitly broken chiral symmetry by baryon density. As noted in [12], kaons condense because the s quark mass is neither too light nor too heavy.

The most complete calculation of possible kaon condensation along the general line described above was carried out by Thorsson et al. [10] with three different $\Sigma_{K N}$, expressed in other terms in their chiral perturbation theory. This calculation contained the medium dependence of the kaon mass through $\Sigma_{K N}$ (see eq. (2)) but did not contain the medium dependence of $f_{\pi}$, the inmedium pion decay constant as required by HLS theory which we believe is indispensable as we will see below. 1

$\overline{1}$ In HLS treatment, this is connected to what is called "intrinsic background dependence" resulting from Wilsonian matching to QCD, the condensates of which 
One may raise objections to the above simple treatment on the ground that several mechanisms that could be important are left out in the treatment. Most obvious are the roles of the $\Lambda(1405)$ and hyperons, much discussed in the literature, which when one does fluctuations from the matter-free vacuum, cannot be ignored. When looked at in terms of an RG flow, these degrees of freedom give "irrelevant" terms for the location of the critical density, that is, insignificant for the precise value but they play a determinant role in triggering phase transitions.

Since the role of the $\Lambda(1405)$ in nuclei and dense matter has been greatly disputed in the past, we clarify what it as well as other related degrees of freedom - such as p-wave hyperon interactions - that we will refer to as "dangerously irrelevant operators" following condensed matter terminology, do in the condensation phenomenon. The point we will drive at is that while they may play a crucial role in driving the system toward instability, once the direction in which the system moves is determined, the location of the critical density is highly insensitive to the strength of such interaction terms involved.

We focus on the contribution of $\Lambda(1405)$ to kaon-nuclear interactions. 2 The $\Lambda(1405)$-nucleon interactions can be written as four-Fermi interactions which have canonical dimension 6. As such, it is "irrelevant" from the usual dimensional counting. To show what such a term does in meson condensation, we follow the argument given in [12] for generic meson condensation in strong interaction physics. It is a toy-model argument but it is applicable to the present case. The technique used is the one developed by Shankar [13] for Landau Fermi liquid theory. There is a distinct difference, however, between boson condensation and Fermi liquid in that while a "mass" term is relevant in the renormalization group sense, so figures importantly in the condensation process, the effective mass in the Fermi liquid is a fixed-point quantity, so it does not flow. This is because the Fermi surface in the latter is fixed by fiat.

Let the meson field be denoted generically as $\Phi$. We will focus on two terms, one "mass" term and the other interaction term, with the action written schematically (apart from the kinetic energy term)

$$
S=S_{M}+S_{I}
$$

where

$$
S_{M}=\int d \omega d^{3} q \tilde{M} \Phi^{*} \Phi,
$$

are background - temperature, density etc. - dependent.

2 Similar arguments can be made for p-wave hyperon interactions. They give rise to "irrelevant" four-Fermi interactions. 


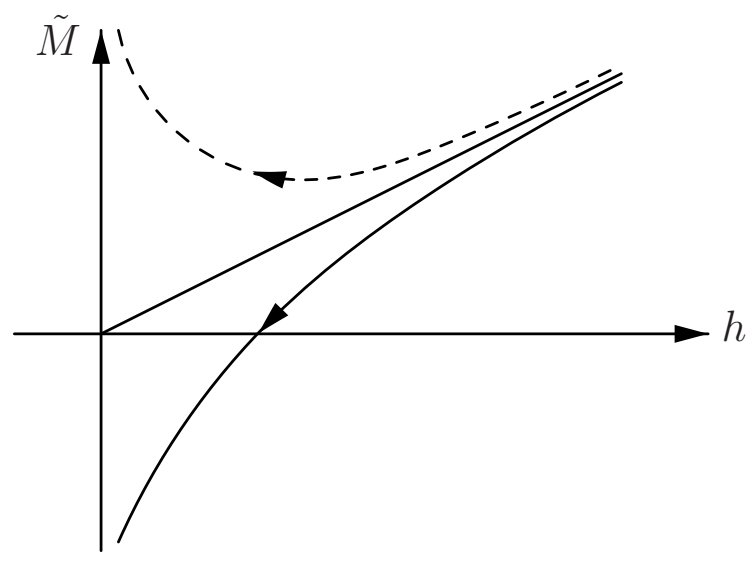

Fig. 2. Schematic diagram of RGE flow for the mass $\tilde{M}$. The dotted line with arrow up shows the flow for $D<\frac{3}{2} \frac{\tilde{M}_{0}}{h_{0}}$ and the solid line with arrow down for $D>\frac{3}{2} \frac{\tilde{M}_{0}}{h_{0}}$.

$$
S_{I}=\int\left(d \omega d^{3} q\right)^{2}\left(d \epsilon d^{3} k\right)^{2} h \Phi^{*} \Phi \Psi^{\dagger} \Psi \delta^{4}(\omega, \epsilon, q, k)
$$

where $\Psi$ is the baryon field containing nucleons, hyperons and $\Lambda(1405)$ etc. Of course both $\tilde{M} 3$ and $h$ subsume many terms with varying powers in the suitable expansion (e.g., the chiral expansion in chiral perturbation theory). 4 For our discussion we need not specify them here.

The scaling law for the fields involved under scaling transformation determined from the kinetic term (which is required to be invariant under the scaling) shows that the "mass" term is relevant but the interaction $(h)$ term is irrelevant. Now the RGEs are found à la Shankar [13] by making the scale change $\Lambda \rightarrow s \Lambda$ with $0<s<1$ where $\Lambda$ is the cut-off scale [12],

$$
\begin{aligned}
\frac{d \tilde{M}}{d t} & =\tilde{M}-D h \\
\frac{d h}{d t} & =-h / 2
\end{aligned}
$$

where $t=-\ln s$ and $D=d n$ with $d>0$ is a constant depending on $\Lambda / k_{F}$ where $k_{F}$ is the Fermi momentum of the baryonic system. The solutions to the RGEs are

$\overline{3}$ Tilde $r e p r e s e n t s$ that it contains other "mass-like" terms.

4 For instance, $h$ will contain, aside from the kaon mass, leading $O(p)$ term of $\sim \frac{\omega}{f_{\pi}^{2}} K^{\dagger} K N^{\dagger} N$, the next-to-leading order $O\left(p^{2}\right)$ terms, $\sim \frac{\Sigma}{f_{\pi}^{2}} K^{\dagger} K N^{\dagger} N$ etc. 


$$
\begin{aligned}
\tilde{M}(t) & =\left(\tilde{M}_{0}-\frac{2}{3} D h_{0}\right) e^{t}+\frac{2}{3} D h_{0} e^{-t / 2}, \\
h(t) & =h_{0} e^{-t / 2}
\end{aligned}
$$

As one decimates down with $s \rightarrow 0(t \rightarrow \infty)$ integrating out higher lying modes, the boson-fermion interaction gets exponentially suppressed. We see that if $D<\frac{3}{2} \frac{\tilde{M}_{0}}{h_{0}}$, then the $\tilde{M}$ flows toward $+\infty$. On the other hand, for

$$
D>\frac{3}{2} \frac{\tilde{M}_{0}}{h_{0}}
$$

the $\tilde{M}$ is bound to turn negative, signalling instability against condensation. This can be seen in Figure 2. Now $D=d n$ and $d>0$, so that means that (16) can be satisfied by dialling the density. What matters is the "least irrelevant term" figuring in $h_{0}$ that drives flow toward condensation while more irrelevant terms - unless of course numerically enhanced - get suppressed.

Once the system, flowing toward instability, reaches the critical point, then what matters will be the least "irrelevant" term that "eats up" the mass, with other irrelevant terms being suppressed. In fact a detailed analysis shows that the $\Lambda(1405)$ that brings the initial attraction to direct the RG flow figures negligibly in determining the location of the critical point [8]: it is found that change of the $\Lambda(1405)$-nucleon interaction strength by orders of magnitude affects the critical density by only a few $\%$.

Another objection that could be raised is that no account is made of the strong repulsion that can be generated in nuclear interactions in dense medium that would prevent kaon condensation at such a low density. In [14], Carlson et al. argue that due account of short-range nuclear correlations could push the critical density above $\sim 7 n_{0}$. This objection will be answered in what follows in terms of "hadronic freedom" in the vicinity of the critical density which prevents the repulsion from growing at high density.

\subsection{Strangeness Condensation By Expanding About the Fixed Point Of the Harada-Yamawaki Vector Manifestation}

As the second estimation of the critical density which we favor over the others, we calculate kaon condensation going top-down from the chiral restoration point. For this we adopt the hidden local symmetry approach to chiral dynamics developed by Harada and Yamawaki (HY) [1]. It is appealing to think of this theory as a truncated infinite-tower description of holographic dual QCD that arises from string theory (explained below) in which all vector mesons lying above the lowest ones, $\rho, \omega$ and $\phi$, are integrated out. This theory is 
characterized by that it has local gauge invariance, which allows a systematic chiral perturbation expansion with the vector mesons included and that the effective theory is matched to QCD à la Wilsonian RGE and hence "knows" about QCD. The crucial outcome of the HY's analysis is that the theory has what is called "vector manifestation (VM)" fixed point to which the theory flows subject to constraints imposed by QCD when the system is driven by either temperature [15], density [16] or the number of flavors [1] to the critical point at which chiral symmetry is restored. The fixed point is given by

$$
p^{*}:=\left(g^{*}, a^{*}, f_{\pi}^{*}\right)=(0,1,0)
$$

where $g$ is the hidden gauge coupling constant, $a=F_{\sigma}^{2} / F_{\pi}^{2}$ is the ratio of "parametric decay constants" of the pion $F_{\pi}$ and the longitudinal component of the $\rho$ meson $F_{\sigma}$ and $f_{\pi}$ is the physical pion decay constant.

What is highly pertinent to us is that near the VM fixed point, fluctuations of the hadrons figuring in the theory become well-defined, making processes taking place near the VM fixed point amenable to a simple calculation. It has been observed that even if a given process takes place away from the VM fixed point, so $g \neq 0$ and $f_{\pi} \neq 0$, taking $a \approx 1$ can be a good starting point for describing fluctuations in certain processes. For example, $a=1$ is an accurate approximation for describing the chiral doublers in heavy-light mesons such as $D$ and $D^{*}[17]$, the $\pi^{+}-\pi^{0}$ mass difference [18], the nucleon EM form factors [19] etc.

Assuming that kaons condense near, but below, the critical density for chiral restoration $n_{\chi}$, we start from the VM fixed point (17) and make a renormalizationgroup flow analysis as density decreases from the VM fixed point as was done in [20]. In performing this calculation, we exploit two observations on: (1) the relevant degrees of freedom near the VM fixed point and (2) the weakness of interaction strength in what is referred to as "hadronic freedom" zone.

We discuss these observations.

The first and most important observation is that the relevant degrees of freedom for kaon condensation are not those manifest in free space where the elementary kaon-nucleon interaction takes place, but more relevant are the degrees of freedom in the vicinity of the fixed point, since the condensation is in the neighborhood of this point $n_{\chi}$ at which chiral restoration takes place. Thus, for example, since $\langle\bar{q} q\rangle$ is near zero, the HLS gauge coupling constant $g$ is near zero, making the vector mesons nearly massless as pions are. This makes chiral perturbation calculation feasible and reliable with the vector mesons and pions treated on the same footing with their masses appearing as small parameters of $O\left(p^{2}\right)$. Furthermore, the $\Lambda(1405)$ and other excitations which are important near the matter-free vacuum, become irrelevant in the sense 
defined above, so do not figure in the calculation of the critical density.

We thus have vector mesons and pions as the explicit degrees of freedom. But then what about baryons?

In holographic QCD which generalizes Harada-Yamawaki's HLS theory, there are no fermion degrees of freedom. Baryons therefore must arise as solitons. Indeed in the holographic dual QCD model of Sakai and Sugimoto [21] which has correct features of chiral symmetry properties of QCD proper, baryons do arise naturally as instantons in five dimensions in which low-energy QCD is dualized or alternatively as skyrmions in four dimensions embedded in an infinite tower of vector mesons. The chiral properties of the resulting baryons, in particular, nucleons, are very well described in the approximations adopted by the model [22]. In order to exploit the VM fixed point, we need to integrate out all except the lowest members of the tower and treat hadonic matter in terms of skyrmions emerging from HY's HLS theory. Indeed hadronic matter at high density has been described in terms of skyrmion matter with an infinite winding number [23]. However there is an indication that skyrmions are not stable at some density $n_{\text {flash }}$ above $n_{0}$ [24]. We will argue that this implies that the nucleon goes over to constituent (or quasi) quarks at $n_{\text {flash }}<n_{\chi}$. We will first develop the argument in temperature and then apply it to the density case.

\subsection{Hadronic Freedom, the Flash Point And Why the Constituent Quark Model Is Relevant}

What happens when hadronic system is heated to near the critical temperature $T_{\chi}$, i.e., from 120 to $175 \mathrm{MeV}$, was studied by Brown et al [25] using the STAR peripheral data [26]. For the peripheral experiments $120 \mathrm{MeV}$ was the freezeout temperature; i.e., the hadrons leaving the system at that temperature did not interact further. The upper temperature $T=175 \mathrm{MeV}$ was taken to be the phase transition temperature $T_{\chi}$ at which the VM dictates that the HLS coupling $g$ go to zero and the parameter $a$ go to 1 . Brown et al. [25] showed that the widths $\Gamma(\rho \rightarrow 2 \pi)$ for $\rho$ decay into two pions were zero at $T=T_{\chi}$ and remained small until just before freezeout at $T=120 \mathrm{MeV}$. This was because the $\Gamma^{\prime}$ 's went as $\left(m_{\rho}^{\star} / m_{\rho}\right)^{5}$ and the mass of the $\rho$ was taken to increase from zero at $T_{\chi}$ to $90 \%$ of the on-shell $\Gamma$ at $120 \mathrm{MeV}$. The latter value was called the "flash point" because the interactions of the $\rho$ 's at that temperature was that of (nearly) on-shell hadrons. The $\rho$-mesons in the experiment were recreated by following the pion tracks in the time projection chamber back to the vertex, identified as the $\rho$, for those which were emitted in $\rho$-decay. The $120 \mathrm{MeV}$ was thus not only the freezeout temperature, but also the temperature at which the hadrons turned into constituent quarks. 
We learn from the above observation that the region of temperature from $\sim 120$ to $T_{\chi}$ thus delineates a region in which interactions are negligible, a region governed by the vanishing coupling constant $g \sim\langle\bar{q} q\rangle \rightarrow 0$. We can also deduce what happens to the $a$ parameter which is defined by

$$
m_{\rho}^{2}=a F_{\pi}^{2} g^{2} .
$$

As is known by the KSRF relation, $m_{\rho}^{2}=2 F_{\pi}^{2} g^{2}$, and also from the vector dominance of the pion EM form factor, $a=2$ in matter-free space. In the large $N_{c}$ limit in which the bare HLS Lagrangian is defined by Wilsonian matching, one finds $a \simeq 4 / 3$ with the $a=1$ point lying on the RG trajectory matched to QCD. The $a=2$ point is not on that trajectory, implying that in HLS theory, $a=2$ can only be an accident. Indeed as mentioned above, nature prefers $a \approx 1$ at least in the leading order of chiral perturbation theory. It has been shown by Harada and Sasaki [27] that in heat bath, the vector dominance given by $a=2$ is strongly violated as $a$ goes to 1 when temperature goes toward $T_{\chi}$. This suggests that we associate $a_{\text {flash }} \sim 4 / 3$ as the flash point at which $T=T_{\text {flash }} \sim 120 \mathrm{MeV}$. We interpret the region between $a \sim 4 / 3$ at $120 \mathrm{MeV}$ and $a=1$ at $T_{\chi}$ to be a region in which the relevant fermionic degrees of freedom are constituent quarks, with the off-shell quarks flowing freely from higher to lower temperature. Nothing detectable goes on here in this region - it is just a region of space-time without anything except off-shell particles within it, hence called "hadronic freedom" region.

In dense matter, the situation is much less clear, so we are going to make a certain number of guesses and develop a scenario that parallels the temperature scenario. 5

As mentioned, it is established that the vector manifestation fixed point (17) is reached when density is at the critical density $n_{\chi}$ at which $\langle\bar{q} q\rangle=0$ [16]. It has also been verified that $m_{\rho} \rightarrow g \propto\langle\bar{q} q\rangle \rightarrow 0$ in the vicinity of the critical density and $(a-1) \propto(\langle\bar{q} q\rangle)^{2} \rightarrow 0$. However the question is: where is the density flash point above which the system goes into the hadronic freedom region? In order to answer this question within the framework of HLS theory, we need the baryon degrees of freedom and at present, there is no systematic calculation in HLS theory that includes baryons. What we know now is described in [19]: Up to the nuclear matter density $n_{0}$, the available phenomenology in nuclear processes indicates that the HLS coupling $g$ stays more or less unchanged. Beyond $n_{0}$, we have no information from data but our basic assumption is that the flash point $n_{\text {flash }}$ lies at the point from which $g$ drops rapidly arriving at $g=0$ at $n=n_{c}$. From what we discussed above, we deduce that it is at $n_{\text {flash }}$

5 There is an indication from a skyrmion description of dense matter that $a \approx 1$ at the "pseudo-gap density" which is identified as the flash density [28]. This may be a better approximation than $a \simeq 4 / 3$ adopted below. 
that going top-down, the constituent quarks go on-shell forming baryons as the coupling constant becomes strong leaving the hadronic freedom region. Now if one extrapolates linearly from $a=1$ at $n_{\chi}$ to $a=2$ at $n=0$, we arrive at $a \simeq 4 / 3$ when the density reaches the density

$$
n_{\text {flash }} \simeq 3 n_{0}
$$

This is the density counterpart of $T_{\text {flash }} \simeq 120 \mathrm{MeV}$. The hadronic freedom region is identified as the interval $n_{\text {flash }} \lesssim n_{H F} \lesssim n_{\chi}$. The putative repulsion one expects naively from the vantage point of the matter-free vacuum cannot survive within this region and would not provide the mechanism often invoked for stabilizing a neutron star at as large as $\gtrsim 2 M_{\odot}[29] 6$

$\overline{6}$ We can understand this in terms of an effective chiral Lagrangian that incorporates Brown-Rho scaling (or "intrinsic density dependence (IDD)" in HLS theory) [4]. Consider four-Fermi (baryon) interaction terms in the effective Lagrangian relevant to the vector-meson channels:

$$
\sum_{i} C_{i}^{\star}\left(\bar{N} \Gamma_{i} N\right)^{2} \approx-\frac{C_{\tilde{\omega}}^{\star 2}}{2}\left(\bar{N} \gamma_{\mu} N\right)^{2}-\frac{C_{\tilde{\rho}}^{\star 2}}{2}\left(\bar{N} \gamma_{\mu} \tau N\right)^{2}+\cdots
$$

Here the asterisk denotes the IDD (intrinsic density dependence) in the parameters, i.e., Brown-Rho scaling, appearing in the EFT Lagrangian and the ellipsis stands for other terms that are allowed by chiral symmetry. Suppose now that the parameters in the matrix elements of the four-Fermi interactions (20) are expanded in power of the density operator $\hat{n}=\bar{N} \gamma_{0} N$. The resulting Lagrangian will contain six- and higher-Fermi fields and when mean field is taken, will give rise to density dependent parameters in the theory. This means that certain chirally symmetric $n$-body (with $n>2$ ) interactions are subsumed in the IDD of the coefficients in (20) when truncated to the four-Fermi terms. Since the mean field using the Lagrangian is equivalent to doing Landau Fermi liquid theory $[4,30]$, certain important many-body effects are therefore encoded chirally symmetrically in the IDD of the parameters.

In this formulation, the repulsion resides in the $\omega$ channel in (20). In HaradaYamawaki HLS theory, the parametric mass of the $\rho$ and $\omega$ (or the lowest member of the infinite tower in holographic QCD) drops as density increases whereas the vector coupling $g$ remains more or less unchanged up to $n \sim n_{0}$ and then decreases after the "flash density" $n_{\text {flash }} \sim 2 n_{0}$. This means the coefficient $C_{\tilde{\omega}}^{\star 2}$ will increase as $g^{2} / m_{\omega}^{* 2}$ with the falling mass up to, say, $n \sim n_{0}$ or slightly above. This repulsion up to that density provides the mechanism for the saturation of nuclear matter. From $n_{\text {flash }}$, the VM (vector manifestation) starts becoming operative, with the gauge coupling and the mass falling at the same rate so that the ratio $g^{* 2} / m_{\omega}^{* 2}$ will remain constant at higher density. The repulsion will cease accordingly. 


\subsection{Calculation Of the Critical Density $n_{c}$ For Kaon Condensation}

We now have all the pertinent degrees of freedom in the vicinity of $n_{\chi}$. They are the constituent (or quasi) quarks, the (pseudo)Goldstone bosons $\pi$ and $K$ and the vector mesons $\rho$ and $\omega$ with their masses comparable to the pion mass. To describe kaon condensation, we need to take into consideration interactions between the kaon and the quasiquarks. The Lagrangian we have is HLS Lagrangian that is matched to QCD at a density $n \lesssim n_{\chi}$ at a matching scale $\Lambda_{M}^{*}$. Now we need to calculate RGE flow in theory with the gauge coupling $g$ and the parameter $a$ as described above in the density region $n_{\text {flash }} \lesssim n \lesssim n_{\chi}$. The only other quantity that we have to consider is the parametric pion decay constant $F_{\pi}^{*}$, since kaons will couple to the quarks with the constant $1 / F_{\pi}^{*}$. We need this constant at the matching scale $\Lambda_{M}^{*}$. For this we observe that $F_{\pi}^{*}$ does not scale appreciably in the matter-free space as found by Harada and Yamawaki [1]. We shall give an argument why we expect this to be similar in medium. For this, it will suffice to to know what it is near the nuclear matter density. This information comes from the analysis of deeply bound pionic atoms $[31] 7$ from which we learned that the in medium parametric $F_{\pi}$ must be decreased

$$
F_{\pi} \rightarrow F_{\pi}^{*}\left(n_{0}\right) \approx f_{\pi}^{\star}\left(n_{0}\right) \approx 0.8 F_{\pi}
$$

$\sim 20 \%$ at $n=n_{0}$. We have no systematic calculation of $F_{\pi}^{*}$ beyond the nuclear matter density. This is because $F_{\pi}^{*}$ runs with the intrinsic density dependence dictated by the Wilsonian matching of the HLS correlators to those of QCD, which is unknown since lattice techniques cannot handle density effects. However one can argue that it will stay more or less unchanged up to the critical density. In both temperature and density, when one goes beyond the flash point, the vector meson mass $m_{V}^{*}$ and the gauge coupling constant $g^{*}$ scale in the same way, i.e. proportionally to $\langle\bar{q} q\rangle^{*}$. Hence the ratio $g^{*} / m_{V}^{*} \sim 1 / F_{\pi}^{*}$ - with $a^{*} \approx 1$ - will stay more or less unchanged. This feature was exploited in the high-temperature case in [33]. As for the density relevant to kaon condensation, we need not go all the way to the chiral restoration point but one can make a rough estimate of the ratio $F_{\pi}^{*}\left(n_{\chi}\right) / F_{\pi}(0)$ in a HLS model with quasiquarks [16],

$$
F_{\pi}^{*}\left(n_{\chi}\right) / F_{\pi} \sim \sqrt{3 / 5} \approx 0.77
$$

It thus seems justified to take the scaling (21) operative from the flash point to the kaon condensation point. It was shown in [20] that the Walecka vector

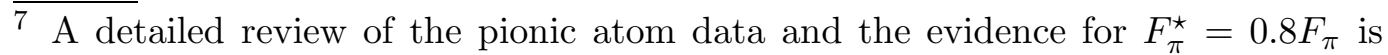
given by E. Friedman and A. Gal [32]. 
mean field used so much in nuclear physics already had this increase in it, so that it is part of the usual phenomenology of nuclear physics.

Since the gauge coupling constant $g$ is near zero in the hadronic freedom region, quantum loop corrections can be ignored. It suffices therefore to do the mean field calculation (i.e., tree contributions). For the neutron fraction $x_{n}$ and proton fraction $x_{p}$ in a compact star, the potential felt by the kaon will then be

$$
V_{K^{-}}=-\frac{1}{a^{*} F_{\pi}^{* 2}}\left(\frac{x_{n}}{2}+x_{p}\right) n .
$$

We have included $\rho$ as well as $\omega$ exchange so as to make a typical neutron star charge up at density $n_{\chi}$. Note that (23) is not in linear-density approximation. In this picture, this is the only important contribution to the effective mass of the kaon. Other terms are suppressed by the hadronic freedom effect. Now let us see how the effective kaon mass behaves near the chiral phase transition density $n_{\chi} \sim 4 n_{0}$. For this we take $a^{*}=1$ and $F_{\pi}^{*} / F_{\pi} \simeq 0.8$. Then we find

$$
\frac{\left[g_{V}^{\star} / m_{V}^{\star}\right]_{\text {fixed point }}}{\left[g_{V}^{2} / m_{V}^{2}\right]_{\text {zero density }}}=\frac{\left[a F_{\pi}^{2}\right]_{\text {zero density }}}{\left[a^{\star} F_{\pi}^{\star}\right]_{\text {fixed point }}}=\frac{2}{0.8^{2}} \simeq 3.1
$$

For $x_{n} \approx 0.9$ corresponding to the neutron-star matter, we have from (23)

$$
V_{K^{-}}\left(n=4 n_{0}\right) \approx-516 \mathrm{MeV}
$$

This suggests that the kaon must have a vanishing mass at the chiral transition density.

Let us move down to the flash point $\sim 3 n_{0}$. At this density, taking into account the flow of $a$, we find

$$
m_{K}^{*}\left(\frac{3}{4} n_{\chi}\right) \frac{4}{3} \simeq m_{K^{-}} / 4 \simeq 165 \mathrm{MeV}
$$

This is somewhat smaller but not too far from the electron chemical potential expected at that density, $\sim 220 \mathrm{MeV}$ [10]. Considering the roughness of the estimate, it seems reasonable to identify the flash point as the kaon condensation critical density $n_{c}$. We note that were it not for the presence of the electron Fermi sea, the flow of the star matter would end up at the VM fixed point at $n_{\chi}$. However, the decay of electrons into kaons stops the hidden local symmetry (HLS) flow. 


\subsection{Kaon Condensation From the Fermi-Liquid Fixed Point}

The third way to arrive at the kaon condensation critical density $n_{c} \sim 3 n_{0}$ is to fluctuate from the density at which nuclear matter is saturated, namely, $n_{0} \approx 0.16 \mathrm{fm}^{-3}$. The basic idea has been presented in previous review articles by the authors, the most recent of which is given in [30]. As discussed there, going above the nuclear matter density consists of first writing an effective Lagrangian that describes nuclear matter as the Fermi-liquid fixed point à la Shankar when the mean field approximation is taken. Given such a Lagrangian

with the parameters determined with the "intrinsic density dependence" as dictated by the Wilsonian matching to QCD prescribed in HLS theory, one can make a fluctuation around the Fermi liquid fixed point to go to higher density. The effective Lagrangian which is anchored on chiral symmetry is equivalent to Walecka's linear mean field Lagrangian with the intrinsic density dependence (i.e. Brown-Rho scaling) suitably taken into account.

To proceed to kaon condensation, we assume as above that the relevant fermionic degrees of freedom are the constituent quarks. We consider the constituent quark picture applicable at nuclear matter density and above and that the nucleon is made of loosely bound three quasiquarks and the kaon of loosely bound strange quark and chiral anti-quasiquark. Now the mean field nuclear potential has two parts, the vector potential $V_{N}$ and the scalar potential $S_{N}$, which can be viewed as due to exchange of an $\omega$ meson and a scalar meson (denoted here $s$ ),

$$
\begin{aligned}
V_{N} & =\frac{9}{8 F_{\pi}^{* 2}} n, \\
S_{N} & =-\frac{g_{s}^{* 2}}{m_{s}^{* 2}} n .
\end{aligned}
$$

Here we are introducing a fictitious scalar in the line of Walecka model with scaling mass and coupling constant but one can actually avoid the scalar degree of freedom by writing the effective Lagrangian in terms of a four-Fermi interaction (see, e.g., [30]). Now from the phenomenology of Walecka model, we know that

$$
S_{N}\left(n_{0}\right)-V_{N}\left(n_{0}\right) \lesssim-600 \mathrm{MeV} .
$$

Taking into account of the fact that the kaon contains only one non-strange quark compared with three in the nucleon, we expect the kaon to feel the attractive potential

$$
S_{K^{-}}+V_{K^{-}} \lesssim-200 \mathrm{MeV} .
$$


Now applying the same reasoning to the compact star matter with a $90 \%$ neutron fraction, we find at $n \sim 2 n_{0}$ that

$$
\omega_{K^{-}}\left(2 n_{0}\right) \sim 160 \mathrm{MeV}
$$

Since the electron chemical potential expected at this density is $173 \mathrm{MeV}$ [10], we see that kaon condensation is expected to take place already at $2 n_{0}$ in this scenario. Thus this picture predicts $n_{c}<3 n_{0}$ consistent with the two other estimates.

\subsection{Delayed Collapse Of Neutron Star}

It is not generally realized that the metastability of SN1987A, i.e. the fact that it emitted neutrinos for about 12 seconds before disappearing (and was "never heard from again"), was probably aided substantially by the presence of strange particles in the core [34]. For this, the fact that the particles were strange was not the important point; the strange particles with strange quarks, be they the $K^{-}$or the hyperon $\Sigma^{-}$, have negative charges and additional protons would be needed to neutralize their charge. The additional protons would interact strongly attractively with the neutron main component in the neutron star and increase the binding energy of the star. As the star collapses to a neutron star, once the densities are $\sim 10^{12} \mathrm{~g} \mathrm{~cm}^{-3}$ the neutrinos are trapped. That is, they may try to random walk their way out, in the frame of the infalling matter, but once the density is $\sim 10^{12} \mathrm{~g} \mathrm{~cm}^{-3}$ the mean free path for neutrino scattering off the nucleons is only a few centimeters, so the neutrino drift (random walk) velocity outwards becomes smaller than the velocity of infalling matter. The neutrinos, above the trapping density, equilibrate with the neutrons and protons so that

$$
\mu_{n}=\mu_{p}+\mu_{e}-\mu_{\nu}
$$

and the neutrinos fill a Fermi sea of Fermi energy $\sim 100 \mathrm{MeV}$. Thus, the neutrinos contribute to the pressure.

It has been considered likely that the neutron star has $\Sigma^{-}$hyperons in it. Although the mass of the $\Sigma^{-}$hyperon is $1197 \mathrm{MeV}$, much heavier than the neutron $940 \mathrm{MeV}$, the neutrons will be degenerate. Since the admixture of $\Sigma^{-}$ will have large negative charge, its presence would bring down the electron chemical potential $\mu_{e}$ considerably [36]. The $\Sigma^{-}$at rest can replace both an electron and a neutron. It begins to come in at a density such that

$$
\mu_{\Sigma^{-}}=\mu_{n}+\mu_{e}
$$


This is generally satisfied at a density $n \sim 2 n_{0}$; i.e., at about twice nuclear matter density [35]. (The $\Lambda$ 's come in earlier, at a lower density, but the $\Lambda$ is charge neutral and does not have the interesting effects that the $\Sigma^{-}$has.) As the neutrinos leave, the electrons recombine with the protons to make neutrons and $\Sigma^{-}$'s, and the EOS stiffens as the number of protons decreases. Thus, the star will not collapse immediately into a black hole. Kaon condensation will set in later as the star cools down further.

As has been discussed extensively in the literature, the appearance of hyperons in dense matter has been invoked to argue against kaon condensation setting in at a density $\lesssim 3 n_{0}$ because the driving force, i.e., the electron chemical potential, would get weakened [36]. We believe this argument is not valid. We have made clear that nucleons and hyperons are no longer the relevant degrees of freedom when the flash point, which we estimate to be at $\sim 3 n_{0}$ is reached. The RG flow is run completely by the Weinberg-Tomozawa vector interaction here, the hyperons having been integrated out, as irrelevant. Our fluctuation about the fixed point rather than about the perturbative vacuum has given a scaling which increases the vector interaction by $\left(a^{\star}\right)^{-1}$, increasing from 0.5 in free space to 1 at the fixed point. This explains much of the increased attraction and, therefore, softening of the EOS in our nonperturbative calculation.

\section{A SCENARIO FOR A LARGE NUMBER OF LOW-MASS BLACK HOLES IN THE GALAXY}

This was the title of a paper written by G.E. Brown and H.A. Bethe in W.K. Kellogg Radiation Laboratory [37]. Since that time, much improvements on the calculations have been made, the most important one of which being the expanding about the VM fixed point of hidden local symmetry. And furthermore more observational data have been accumulated.

In this second half of the paper, we would like to present a chain of astrophysical observations to link the kaon condensation in hadronic matter developed in the first half to the population of neutron stars and black holes. The most pertinent and daring prediction on this issue had been made by Brown [38], who had found the accepted scenario for binary neutron star evolution to be completely wrong. As we shall outline, the standard scenario accepted up to then assumed that the accretion onto the first-born neutron star, while the companion giant evolved and the neutron star and giant went into common envelope, was limited by the Eddington rate and, therefore, negligible. It was noted in [37], after the suggestion by Chevalier [39], that the rate of accretion necessitated going over to hypercritical accretion and that that was sufficient to send the first-born neutron star into a (low-mass) black hole. It was then suggested that the only way to avoid this was for the two progenitor giants 
to burn helium at the same time, which allowed the neutron star to avoid the red giant stage of the companion giant. This point is further developed below.

The Brown scenario had the prediction that the two neutron stars in a binary, which must have burned helium at the same time, should be within $4 \%$ of each other in mass in order to do this. As we shall see, this prediction is fulfilled by the most massive neutron star binaries.

In the case of the less massive double neutron stars, i.e., J0737-3039 and J1756-2251, the pulsars acquire an additional $0.1-0.2 M_{\odot}$ during the helium red giant stage of the companion, which does not take place in the more massive binaries, but aside from this, the pulsar and companion must be within $4 \%$ of each other in mass. The companion in the less massive double neutron stars, i.e., J0737-3039 and J1756-2251 have to burn hotter than those in the more massive ones during the helium burning in order to reach the same central temperature of the more massive ones because the surface to volume ratio is larger in the less massive stars. This means that more energy is lost from the surface. The result of this is that the progenitors of these two less massive stars go through a helium red giant stage as well as (earlier) a hydrogen red giant stage. During the He red giant stage the pulsar accretes $0.1-0.2 M_{\odot}$ of helium. Therefore, the necessary limit of at most $4 \%$ difference between progenitor masses of the pulsar and companion can be somewhat larger if the companion of the pulsar gives an additional 0.1 to $0.2 M_{\odot}$ of helium during the helium shell burning. Note that the helium burning of pulsar and companion progenitors must overlap in time. The helium red giant stage is put into the evolution of J0737-3039 by Willems and Kalogera [40].

We will try to make our arguments for the general physics community. The general reader would benefit from references to a few review papers, where neutron star structure $[41,42,43]$, supernova explosions $[44,45,46]$ and binary evolution $[47,48]$ are discussed.

\subsection{Hypercritical Accretion Onto First-Born Neutron Star During Common Envelope Evolution}

In the study of neutron stars, a key tool used in astrophysics is the acceptance of Eddington accretion as the maximum possible rate. Matter falling on a neutron star is bound to the neutron star gravitationally by $\sim 20 \%$ of its rest mass because of the great mass of the neutron star. This binding energy is used to produce radiation, say in the form of photons, which are emitted from the surface of the neutron star. These photons scatter off the incoming matter; which we take to be hydrogen, and deposit their momentum in it, by Thomson scattering. At the Eddington limit 


$$
\dot{M}_{\mathrm{Edd}}=R_{6} 1.5 \times 10^{-8} M_{\mathrm{NS}} \mathrm{yr}^{-1}
$$

where $R_{6}$ is the radius of the neutron star in units of $10^{6} \mathrm{~cm}$ (the latter being a typical neutron star radius) and $M_{\mathrm{NS}}$ is the mass of the neutron star in units of the mass of our sun $M_{\odot}$, the outward pressure from the scattering of photons by the hydrogen is sufficient to counteract the inward gravitational force. This, then, gives a maximum rate of accretion in the neighborhood of rates $\sim \dot{M}_{\text {Edd }} . \dot{M}_{\text {Edd }}$ is the rate of mass accretion which just holds off the accreting protons,

$$
\dot{M}_{\text {Edd }}=2 \times 10^{38} \mathrm{ergs} / \mathrm{sec} .
$$

(We shall see later on that accretion can begin again at $\dot{M} \sim 10^{4} \dot{M}_{\text {Edd }}$, where it is called "hypercritical accretion.")

Now the processes that we are going to discuss such as common envelope evolution take a time $\sim$ years. Given the $10^{-8}$ in Eq. (33), accretion at the order of the Eddington rate is unimportant and can be neglected.

Let us begin our evolution of binary neutron stars starting from two giants, with zero age main sequence (ZAMS) masses 16 and $12 M_{\odot}$. Each star burns hydrogen for $\sim 90 \%$ of its lifetime, but the lifetime of the $16 M_{\odot}$ star is shorter than that of the $12 M_{\odot}$. When the former has finished core burning of hydrogen, it expands in red giant stage, and the outer part of the red giant crosses the Roche Lobe, the line of equipotential between the two stars. The more massive $16 M_{\odot}$ star evolves first into red giant transferring $\sim 8 M_{\odot}$ to $12 M_{\odot}$ star by Roche Lobe overflow leaving the He core and $20 M_{\odot}$ star as in Fig. 3.

In binary evolution usually a flat $q\left(q=M_{2} / M_{1}\right)$ distribution in the IMF (initial mass function) is assumed. This has the correlation between primary and secondary star in it that the former is more massive than the latter, which must be present because it evolves first. Although offhand this looks like a quite minimal correlation, it produces factor $\sim 2$ difference in results.

We want to zero in on the common envelope evolution of the first born neutron star with the helium core of the $20 M_{\odot}$ star. The common envelope evolution takes only $\sim$ years, so thinking of Eddington limit for mass accretion of $1.5 \times$ $10^{-8} M_{\odot} \mathrm{yr}^{-1}$ the accretion is quite negligible and has generally been neglected in the literature to date. However it is unreasonable to assume the Eddington rate to hold forever, as the rate of accretion continuously increases. Ultimately the density of matter falling onto the neutron star must be sufficiently high that the photons originating from the neutron star by random walk in the framework of the infalling matter actually move out at a lower velocity than the infalling matter has in falling onto the neutron star, so that the photons are swept back onto the neutron star by the adiabatic inflow. Good discussions 

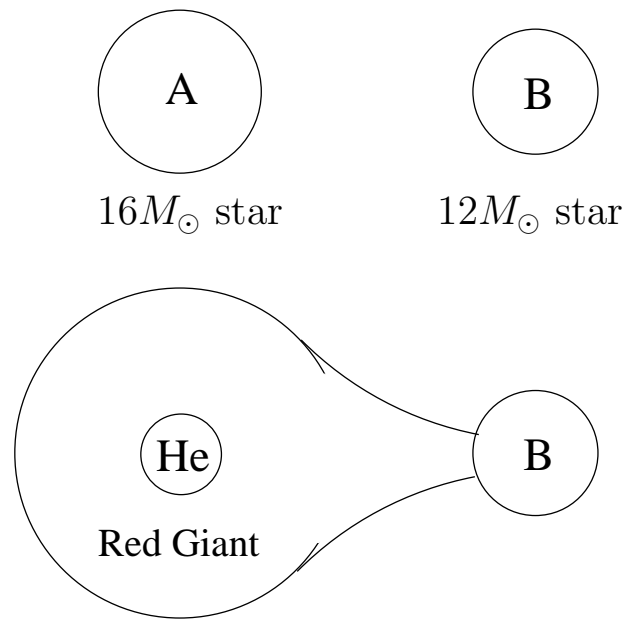

Roche Lobe overflow
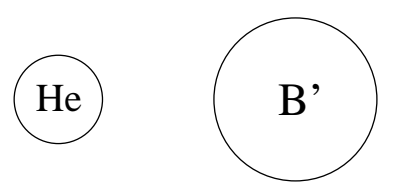

He core $\quad 20 M_{\odot}$ star

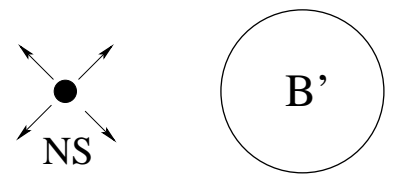

Collapse of He star into a neutron star

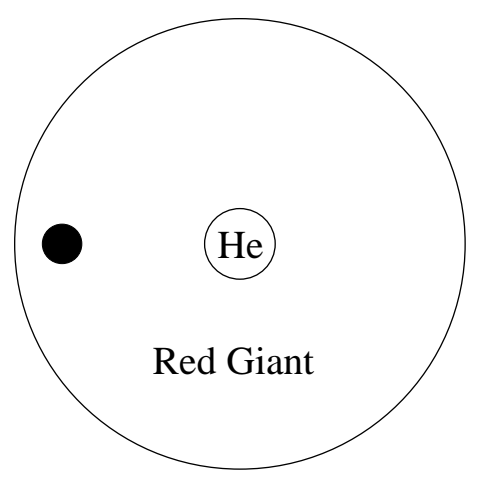

$20 M_{\odot}$ star evolves into red giant, enveloping the neutron star

Fig. 3. Conventional model for double neutron star binary evolution. The neutron star goes into common envelope evolution, dropping towards the star, the increased gravitational binding energy being used to expel the hydrogen envelope. The He star explodes into a neutron star and if the two neutron stars remain bound, this is a binary pulsar. 
of this are given by $[49,50,51,52]$.

With the increase in the rate of accretion, which we scale with Eddington

$$
\dot{m}=\frac{\dot{M}}{\dot{M}_{\mathrm{Edd}}},
$$

in the region of rates $\dot{m} \sim 1$, the cutting off of increase in accretion above that value sets in. The accretion from $\dot{m} \sim 1$ to $10^{4}$ may be complicated, the effects from the Eddington limit causing uneven or sporadic flow, but for $\dot{m}>10^{4}$ where hypercritical accretion sets in, the accretion again becomes simple with uniform flow.

The nature may not be so simple because the matter is accreted at a low temperature, which means that it cannot get rid of its energy rapidly by neutrino emission, which increases with a high power of temperature $T$, but is low for the accretion temperatures.

In the scenario of Bethe and Brown [56], neutrinos were trapped in the collapse of large stars, so the trapping of photons in order to give hypercritical accretion seemed self-evident. Indeed, one can work out that as the accretion increases to the Eddington limit, the photon trapping radius moves to the radius of the neutron star $R_{\mathrm{NS}} \sim 10^{6} \mathrm{~cm}$. There is, then, a range of $\dot{M}$ 's,

$$
\dot{M}_{\text {Edd }}<\dot{M}<10^{4} \dot{M}_{\text {Edd }}
$$

in which the outcome of accretion is not clear, some matter being accepted by the neutron star and some matter being lost in space. This dees not really concern us because for rates $\sim 10^{4} \dot{M}_{\text {Edd }}$ and higher, the accreted matter piles up in an accretion shock. As the amount of matter increases, the density at the base of the accretion shock increases and ultimately, when the temperature reaches $T \sim 1 \mathrm{MeV}$ neutrino pairs carry off the energy as rapidly as it is accreted, holding the temperature to $\sim 1 \mathrm{MeV}$. An analytical calculation of this was carried out by Brown and Weingartner [50].

Given the two giant progenitors going through the evolution shown in Fig. 3, and the helium stars, the lowest mass in the problem was the neutron star mass $M_{\mathrm{NS}}$, and the equations could be solved algebraically if $M_{\mathrm{NS}}$ was set equal to zero [56]. The result was that the first-born neutron star (see Fig. 3) accreted $1 M_{\odot}$ in the common envelope evolution during the red giant phase of the remaining giant. Belczynski et al. [53] (denoted as BKB) removed the approximation made by Bethe and Brown and obtained the result that the accretion was $\sim 0.8 M_{\odot}$ for the lower mass neutron stars and $\sim 0.9 M_{\odot}$ for the higher mass ones. In either case it was sufficient to send the primary into 
a black hole (see Case D2 of BKB). Zeldovich et al. [54] had found already in 1972 that the accreting matter piles up, pushing its way up through the accretion disk. At this stage we go over to the two-dimensional hydro calculations of Armitage and Livio [55]. They showed that an accretion disk reformed inside of the accretion shock, allowing matter to accrete onto the neutron star. They, however, suggested that jets might drive the hypercritical accreting matter off, saving the neutron star from going into a black hole. We find, however, in agreement with observations, that the first born neutron star (the pulsar) is within $4 \%$ difference from the mass of the companion in the binary, in agreement with our scenario that hypercritical accretion can be avoided if the two giant progenitors are within $4 \%$ of each other in mass. No sign of any other amount of hypercritical accretion, which could simply add

to the mass of the neutron star is seen. If, indeed, the accretion were stopped at some intermediate stage by jets, one would expect a distribution in pulsar masses compared with companion masses that differed from the $4 \%$. Since no such distribution is seen, our assumption that the pulsar has gone into a black hole (if the giant progenitors differ by more than $4 \%$ in mass) is validated.

Bethe and Brown [56] found that the accretion was $\sim 10^{8}$ to $10^{9}$ times Eddington accretion, and assumed that with this vast amount of matter, one was back to the classical accretion of Bondi [57] which took no note of the above complication, but did guide the matter correctly through the sonic point; i.e., enforced the classical requirements for accretion. The accretion rate could not be determined directly in the Bethe and Brown [56] calculation, but it was compared with the fully three-dimensional numerical calculation of Terman et al. [58]

\subsection{Formation Of Double Neutron Star Binaries}

We do see double neutron star binaries, so the first-born neutron star cannot always go into a black hole. If the two giant progenitors are so close in mass that they burn helium at the same time, then they would go into a helium common envelope evolution, the one star sending He across the Roche Lobe into the other and the other one sending its expanding helium to the first star [38]. Helium burning takes up $10 \%$ of the star lifetime, most of the time being spent in the main sequence hydrogen burning. To go from lifetimes to masses one must divide by about 2.5, so the two giant progenitors must be within $4 \%$ of each other in mass.

This was a definite prediction modulo one assumption: That helium is not accreted during the helium common envelope evolution. The assumption was justified however by Braun and Langer [59] who showed that the helium burning time is too short for appreciable mass to be accepted by either helium star. 
Table 1

Compilation of NS-NS binaries [60].

\begin{tabular}{lllc}
\hline Object & Mass $\left(M_{\odot}\right)$ & Companion Mass $\left(M_{\odot}\right)$ & References \\
\hline $\mathrm{J} 1518+49$ & $1.56_{-0.44}^{+0.13}$ & $1.05_{-0.11}^{+0.45}$ & {$[61]$} \\
$\mathrm{B} 1534+12$ & $1.3332_{-0.0010}^{+0.0010}$ & $1.3452_{-0.0010}^{+0.0010}$ & {$[61]$} \\
$\mathrm{B} 1913+16$ & $1.4408_{-0.0003}^{+0.0003}$ & $1.3873_{-0.0003}^{+0.0003}$ & {$[61]$} \\
$\mathrm{B} 2127+11 \mathrm{C}$ & $1.349_{-0.040}^{+0.040}$ & $1.363_{-0.040}^{+0.040}$ & {$[61]$} \\
$\mathrm{J} 0737-3039 \mathrm{~A}$ & $1.337_{-0.005}^{+0.005}$ & $1.250_{-0.005}^{+0.005}(\mathrm{~J} 0737-3039 \mathrm{~B})$ & {$[62]$} \\
$\mathrm{J} 1756-2251$ & $1.40_{-0.03}^{+0.02}$ & $1.18_{-0.02}^{+0.03}$ & {$[63]$} \\
\hline
\end{tabular}

Thus, the clear prediction is that two neutron stars in a binary must be within $4 \%$ of each other in mass.

There is, however, one proviso. That is that the lower mass neutron stars in the double pulsars J0737-3039 and J1756-2251 go through not only the hydrogen burning red giant but also a helium burning red giant. The temperature for helium burning in low mass stars must be greater than in higher mass stars in order to ensure a sufficiently high central temperature because of the energy loss through the surface. During the helium burning red giant, $\sim 0.1$ to $0.2 M_{\odot}$ can be deposited on the first born neutron star by the helium star companion, and the first born neutron star should be that much more massive than the other, in addition to the possible $\sim 4 \%$ difference in mass because they must burn helium at the same time.

In Table 1 we show the compilation of masses of double neutron star binaries. In J1518+49 the uncertainties are too large to say anything, except that the largest error $+0.45 M_{\odot}$ is in the direction of equalizing the masses. In the next three binaries the masses are very close. In fact, B2127+11 is in the globular cluster and often considered to have resulted from the exchange of neutron stars formed in different binaries. However, they do satisfy our nearly equal mass scenario. In the evolutionary calculations of the double pulsar J0737-3039A,B the helium red giant phase has been put in $[64,65]$. The $0.1-$ $0.2 M_{\odot}$ accretion during the He red giant phase was calculated in hypercritical accretion by Lee et al. [66]. Using a flat distribution for the Initial Mass Function, Lee et al. calculated in hypercritical accretion what the masses of primary and secondary neutron stars would be in the scenario shown in Fig. 4 in which case the primary (first-born) neutron star is assumed not to go into a black hole, but the neutron star of mass it ends up with after accretion is assumed to be stable. One sees that the primary star, the pulsar, has high $\sim 84 \%$ probability of ending up with mass between 1.8 and $2.3 M_{\odot}$. This is not seen at all in Table 1, so we assume that these must all have gone into black holes. 


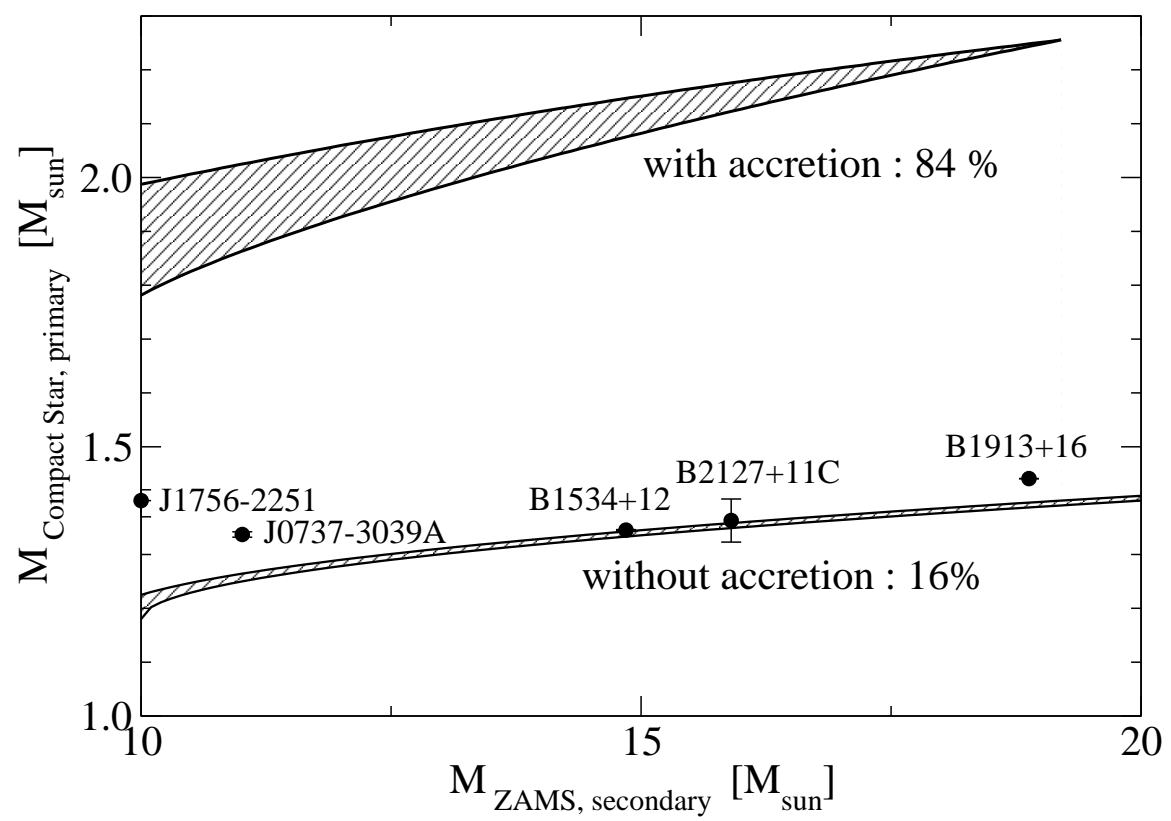

Fig. 4. Masses of primary compact stars with and without accretion during $\mathrm{H}$ red giant stage of secondary star [66]. The filled circles show the primary (more massive) pulsar masses in double neutron star binaries. The corresponding secondary ZAMS masses were obtained from the secondary (less massive) pulsar masses using eq. (8) of Lee et al. [66]. Note that the $84 \%$ corresponds to $M_{\text {Compact Star,primary }}>1.8 M_{\odot}$. There is uncertainty in the final primary compact star masses due to the extra mass accretion, $\sim 0.1 M_{\odot}-0.2 M_{\odot}$, during He giant stage. This may increase the primary compact star masses for both 'with-' and 'without-accretion'. Note that with our maximum neutron star mass of $1.8 M_{\odot}$, all primary compact stars with accretion would go into low-mass black holes.

Note that only the primary pulsar masses (the first born pulsar in the double pulsar) would be affected by the accretion, the companion masses lying on the lower thin band in Fig. 4. In the figure, we have added the primary pulsar masses of 5 double neutron star binaries in filled circles. We left out J1518+49 because of the large uncertainty in its masses. We see that the à priori probability for the primary pulsar masses to lie on the lower thin band within $0.2 M_{\odot}$ uncertainty due to the possible accretion during the He giant phase, given no other possibility than to remain neutron stars, is

$$
P=(0.16)^{5} \simeq 1 \times 10^{-4}
$$

Obviously there is negligible probability assuming random neutron star masses that the two neutron stars in each of 5 binaries are all within $4 \%$ of each other in mass. 
Table 2

Neutron star masses in neutron-star, white dwarf binaries in which the central value of the neutron star mass is $\geq 1.5 M_{\odot}[67]$.

\begin{tabular}{cc}
\hline Name & Pulsar Mass $\left(M_{\odot}\right)$ \\
\hline J1713+0747 & $1.54_{-0.10}^{+0.007}$ \\
B1855+09 & $1.57_{-0.11}^{+0.12}$ \\
J0751+1807 & $2.10_{-0.20}^{+0.20}$ \\
J1804-2718 & $<1.70$ \\
J1012+5307 & $1.68_{-0.22}^{+0.22}$ \\
J0621+1002 & $1.70_{-0.29}^{+0.12}$ \\
J0437-4715 & $1.58_{-0.18}^{+0.18}$ \\
J2019+2425 & $<1.51$ \\
\hline
\end{tabular}

For our later argument, we note here that the Hulse-Taylor pulsar B1913+16 has mass $1.4408 \pm 0.0003 M_{\odot}$. It is the most massive - and the most accurately measured - of the neutron stars in binaries.

\subsection{Neutron Star - White Dwarf Binaries}

In Table 2, we list the neutron-star, white-dwarf binaries in which the central value of the neutron star mass is $\geq 1.5 M_{\odot}$. We should point out from the neutron star masses in Table 2, given the errors noted, that there is no neutron star mass other than in J0751+1807, which is definitely above $1.6 M_{\odot}$. The interval from $1.5 M_{\odot}$ to $2.1 M_{\odot}$ of $0.6 M_{\odot}$ is about half of the total spread, from $1.1 M_{\odot}$ to $2.1 M_{\odot}$ in measured neutron star masses, so it would appear strange for only one of them to lie in the upper half of the possible interval.

On the other hand, sufficient of the central values of the neutron stars in binaries with white dwarf lie above $1.5 M_{\odot}$ that it might be prudent to quote the maximum neutron star mass as the Bethe and Brown [68] value of $1.56 M_{\odot}$ deduced from the cut in the Fe production in 1987A, rather than the earlier $1.5 M_{\odot}[37]$.

The conclusion of Lee et al. [66] is that the maximum neutron star mass must be less than $1.8 M_{\odot}$, otherwise the spectrum of double neutron star binaries would be completely different from that shown in Table 1.

The largest measured mass is in J0751+1807, a neutron star in a binary with white dwarf, with mass $2.1_{-0.20}^{+0.20} M_{\odot}[69]$. However at $95 \%$ confidence level, the mass is $2.1_{-0.5}^{+0.4} M_{\odot}$ so it could be as low as $1.6 M_{\odot}$ at this level. Clearly it could be $1.8 M_{\odot}$ with not unreasonable probability. As mentioned below, the 
measured value has recently been reduced to $1.26_{-0.12}^{+0.14} M_{\odot}$.

A number of calculations were made to estimate the mass of the compact object in SN1987A. They were all more or less in the region of masses arrived at by Bethe and Brown [68] from the $\sim 0.075 M_{\odot}$ of $\mathrm{Ni}$ which was ejected in SN1987A. This gave an estimate of the separation in the density, between the mass that fell back onto the compact object and that which was ejected. The deduced compact object mass was $<1.56 M_{\odot}$. Calculations by Thielemann et al. [70] estimated the mass of the compact object formed in SN1987A to be within the range of gravitational masses $1.30-1.50 M_{\odot}$. The higher values follow from accretion during the formation of the delayed shock. The delayed scenario is now believed to be the correct one. The $1.5 M_{\odot}$ pertaining to SN1987A is supported by the work of Timmes et al. [71].

Tauris and Savonije [72,73] evolved neutron star white-dwarf binaries, mostly by conservative mass transfer, so the evolution should be reliable. Most of their neutron star masses were in the neighborhood of $2.1 M_{\odot}$ whereas all of the other 8 accurately measured neutron star masses in binaries with white dwarfs [60] are close to $1.5 M_{\odot}$. There must be some reason that limits them but we do not know what it is at the moment.

We are well aware that neutron stars in binaries in some globular clusters have been reported with masses substantially above our $1.56 M_{\odot}$ limit. For example, the relativistic periastron advance for the two eccentric systems in the globular cluster Terzan 5, I and J, indicates that at least one of these pulsars has a mass of $1.68 M_{\odot}$ at $95 \%$ confidence [74], not so different from the $1.6 M_{\odot}$ of the Nice et al. J0751+1807 $1.6 M_{\odot}$ at $95 \%$ confidence.

The situation in globular clusters is very different from Galactic. With very low metallicity, their stars resemble more Population II stars than Galactic ones. This should not make a difference however in our calculation of kaon condensation and its consequences since we find the condensation depends only on the baryon number density.

Our statement on kaon condensation is admittedly a very strong one. But it is a falsifiable prediction and should be straightforward to prove us wrong: find a well measured double neutron star binary in which the two neutron stars are more than $4 \%$ different from each other (modulo some small additional shift by He red giant) in mass.

\subsection{Are There Two Branches of Neutron Stars ?}

If the $2.1 M_{\odot}$ neutron star of Nice et al. [69] were firmly established, then there would have to be a loop-hole or an alternative to the Brown-Bethe scenario 
for the formation of neutron stars and light-mass black holes. Indeed Haensel et al. [75] have proposed an interesting way to reconcile the Brown and Bethe $M_{\mathrm{NS}}^{\max }=1.5 M_{\odot}$ with the Nice et al. [69] $2.1 M_{\odot}$ neutron star, two branches of neutron stars - reconciling a $2 M_{\odot}$ pulsar and SN1987A. Two scenarios of neutron star formation were considered. Here we interpret the two scenarios in terms of two possibilities in our language. In the first scenario the formation of the strangeness core is rapid; this is the case of the double neutron star binaries in which the hypercritical accretion from the evolving companion onto the first-born neutron star, the pulsar, is at a rate of $\sim 10^{8} \dot{M}_{\mathrm{Edd}}$, so that the total accretion time is $\sim 1$ year. In the second scenario the neutron star is already present when the main sequence progenitor of the white dwarf begins to evolve. The neutron star accretes matter (and is spun up) by accretion from the companion red giant in stable mass transfer. The rate of mass transfer is $\lesssim \dot{M}_{\text {Edd }}$, so it is $\sim 10^{8}$ times slower than in the first case. Models of quark deconfinement (producing quark matter) are found in which the condition $M_{\mathrm{NS}}^{\max } \gtrsim 2 M_{\odot}$.

The Haensel et al. reconciliation is ingenious and deserves to be paid attention. However in view of the recent development on Nice et al's $2.1 M_{\odot}$ neutron star discussed in the next subsection, reconciliation of that type does not seem called for. We would nonetheless suggest that the reasoning for the two tracks of Haensel et al is not valid for the case at hand. Of the two possible phases, quark matter and kaon condensation, the former phase would be favored at high temperature, as is found in collapses like 1987A, and the latter in a cool scenario like the $\sim 10^{8} \mathrm{yr}$ accretion from an evolving main sequence star onto the neutron star to make a neutron-star, white-dwarf binary. In 1987A the collapsing star heats up, to a temperature $\sim 25 \mathrm{MeV}$ in the center, because of Joule heating. The latter is accomplished by the $\sim 100$ $\mathrm{MeV}$ highly degenerate neutrinos leaving their degeneracy energy in the center of the forming neutron star as they leave with energies determined by the surface temperature. On the other hand, the temperature from accretion onto neutron stars in the white-dwarf, neutron-star binary evolution is much less than $1 \mathrm{MeV}$, so the thermal neutrino emission is almost negligible. Thus, the collapse into strangeness condensation would be favored by the more massive of the two stars in a double-track scenario and the transition into a black hole by strangeness condensation in the collapse of a large star would favor quark matter. In other words, the two tracks of Haensel et al would be filled in the different way, if indeed they were.

\subsection{New Measurement Of the Neutron Star Mass in J0751+1807}

A new value for the mass of J0751+1807 announced by D. Nice in the McGill meeting on "40 Years of Pulsars," August 12-17, 2007, http://www.ns2007.org, 
is now $1.26_{-0.12}^{+0.14} M_{\odot}$ to replace the old value $(2.1 \pm 0.2) M_{\odot}$. This removes the necessity for two branches of neutron stars, with which we had troubles noted just above and reinforces the argument given in [76] that the $2.1 M_{\odot}$ must be wrong, for were it correct, the spectrum of neutron-star masses would be completely different from what it is. Thus, our statement is that all ends with baryon number density $\sim 3 n_{0}$, there is only "nothingness," the space-time volume of the black hole, beyond. Assuming that we are correct, this leads us to the strong statement that color superconductivity, color-flavor locking and all of the other "exotic" phases that have been suggested for high density matter lie in the "black-hole nothingness," being inaccessible by the observers.

\subsection{Neutron Stars In Globular Clusters}

We have no doubt that neutron stars with masses above our limit of $M_{N S}(\max )=$ $1.56 M_{\odot}$ will continue to be reported for globular clusters. 8 No real evolutions of these have been carried out. From the measurement of the rate of advance of the angle of periastron of the binaries combined with matching white dwarfs with the data base, a Bayesian-type analysis gives the possibilities of different white-dwarf masses. Together with the mass function, probabilities of neutron star masses are then obtained.

Binaries with measurable quantities, i.e., those with rapid advances in the rate of periastron advances, are clearly favored and the probability of measurability should be built into the Bayesian analysis.

Why we were so worried about J0751+1807 was that the Shapiro effect was involved in the analysis. With an accurate Shapiro effect and mass function, the two masses in the binary can be measured accurately. The Shapiro effect is a relativistic effect resulting in the time retardation of radiation from the companion passing by the neutron star. Thus, it can only be measured when the angle of incidence is $\gtrsim 80^{\circ}$, so that the neutron star and companion are nearly in the same plane. The measurement of this shift gives a relation between that of the two masses additional to that of the mass function, so with the two relations one can obtain both masses.

Stars in globular clusters have generally low metallicity, more like Population II and III stars. They tend to be much more massive than Galactic stars. Our assertion that kaon condensation with subsequent collapse into black holes depends only on the baryon number density will be very interesting to test in the new environment of globular clusters. Following the present preliminary

\footnotetext{
8 They will surely generate - as the $2.1 M_{\odot}$ mass did - a large number of theoretical publications that will purport to invalidate the onset of kaon condensation at $\sim 3 n_{0}$ and its crucial role in the fate of compact stars.
} 
"Schadenfreude" of observers trying to prove that theorists are wrong we look forward to real tests of our assertion in environments with low metallicity.

\subsection{Convective Carbon Burning And Compact Star Masses}

Although the star in the center of SN1987A disappeared, we do know that the original star Sanduleak-69 202 was in star catalogs and did have a mass of $\sim 18 M_{\odot}$. For many years there was substantial uncertainty in the evolution of giants in the region of masses in which they would begin, with increasing ZAMS mass, to go into black holes. The most important nuclear process in this evolution was the

$$
{ }^{12} \mathrm{C}(\alpha, \gamma){ }^{16} \mathrm{O}
$$

reaction. This reaction determines where the convective burning of carbon stops. In many years of work, the Stuttgart group [77] and more recently Buchmann and Barnes [78] determined this to be

$$
S_{\mathrm{tot}}^{300}=\left\{\begin{array}{ll}
162 \pm 39 \mathrm{keV} \text { barns } & (\text { Stuttgart }) \\
80_{-20}^{+20}+53_{-18}^{+13} \mathrm{keV} \text { barns } & \text { (Buchmann \& Barnes) }
\end{array} .\right.
$$

The upper superscript 300 means that its value is given for a reaction energy of $300 \mathrm{keV}$. The convective ${ }^{12} \mathrm{C}$ burning carries off a lot of entropy through neutrino pairs; it is the first reaction in the stellar burning of stars to do so. Once the ${ }^{12} \mathrm{C}$ is sufficiently used up so that this reaction no longer takes place, the increase in entropy must come from the increase in size of the Fe core, which has entropy $\sim 3 / 4$ per nucleon (a bit less than the $\sim$ unity of the original Bethe et al. paper [79]). We show in Fig. 5 that the convective ${ }^{12} \mathrm{C}$ burning ends at $18 M_{\odot}[80]$. Note that the Fe core mass increases above $1.5 M_{\odot}$ just at $\sim 18 M_{\odot}$. (Brown et al. point out that the Fe core mass should be a good indicator of the compact object mass because the decrease in mass from the general relativistic effect is compensated for by fallback in formation of the compact object.) Thus, with the best available nuclear physics to date, the supernova progenitor of SN1987A collapsed into a compact object of $\sim 1.5 M_{\odot}$, not far from the mass constructed by Bethe and Brown from the $0.075 M_{\odot}$ of $\mathrm{Ni}$ formed in the explosion of $<1.56 M_{\odot} .9$

9 The standard reply of astronomers to the statement that if a neutron star were present in the region where SN1987A exploded, then one should have seen something has been: "The absence of evidence is not evidence of absence." We do not believe this to be true because one would have seen something had a neutron star been there. It could not have just disappeared, leaving no evidence. 


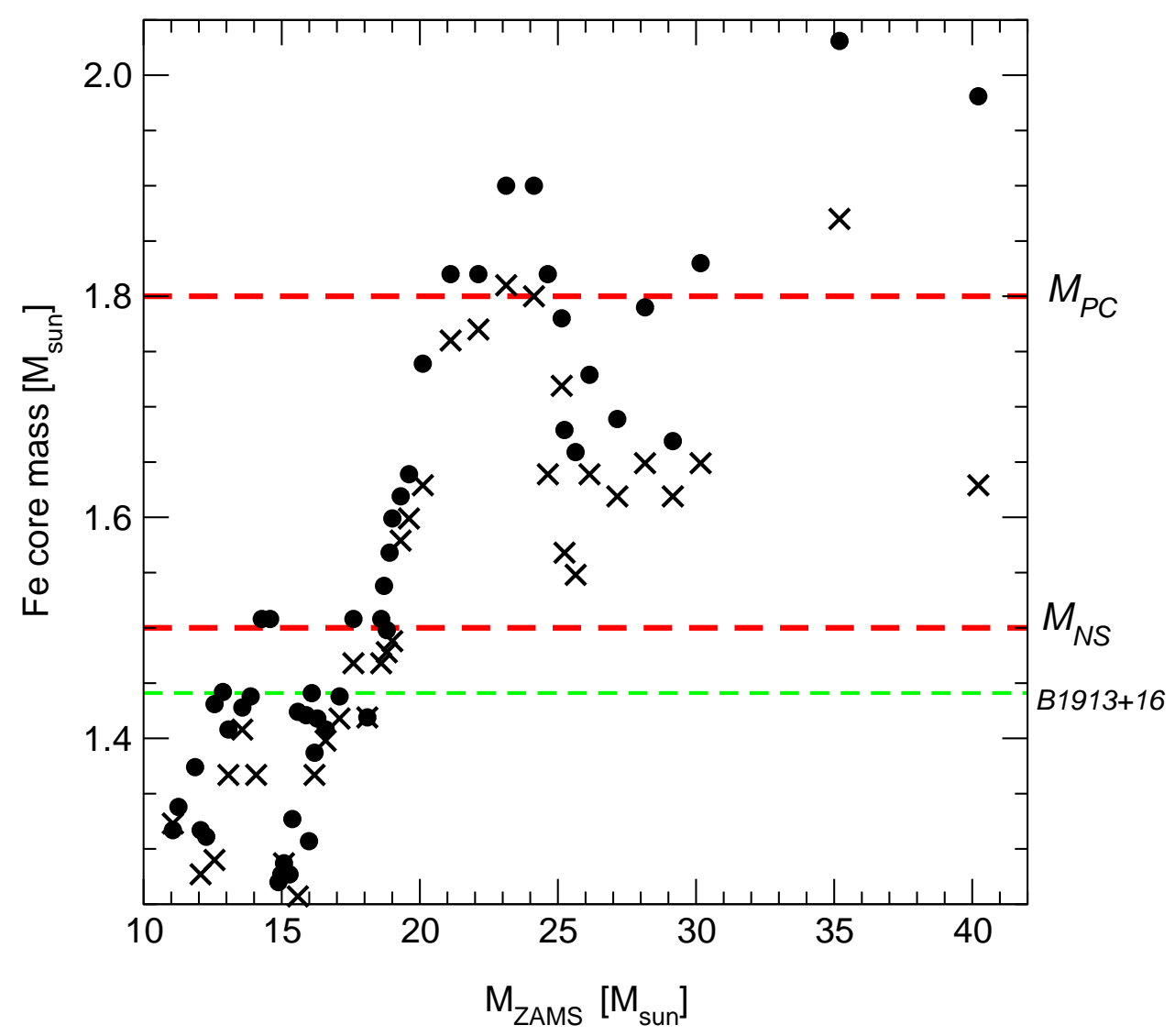

Fig. 5. Comparison of the iron core masses at the time of iron core implosion for a finely spaced grid of stellar masses [81]. The circular black dots were calculated with the Woosley \& Weaver code [82], whereas the crosses employ the vastly improved Langanke, Martinez-Pinedo rates [83] for electron capture and beta decay. If the assembled core mass is greater than $M_{\mathrm{PC}}=1.8 M_{\odot}$, where $M_{P C}$ is the proto-compact star mass as defined by Brown \& Bethe [37], there is no stability and no bounce; the core collapses into a high mass $\mathrm{BH} . M_{N S}=1.5 M_{\odot}$ denotes the maximum mass of NS [37]. The mass of the heaviest known well-measured pulsar, PSR B1913+16, is also indicated with dashed horizontal line [84].

Note that the low-mass black holes will only be formed from ZAMS $\sim 18-$ $20 M_{\odot}$ stars; i.e., black holes of $1.5-2.5 M_{\odot}$. In fact, the black hole from $1987 \mathrm{~A}$ is our only example of such a black hole. The next lowest mass one is the $\sim 5 M_{\odot}$ black hole in the transient source GRO J1655 which comes from an $\sim 30 M_{\odot}$ giant [85]. We would expect black holes from giants with ZAMS masses $20-30 M_{\odot}$ to appear in gamma ray bursts, one of $\sim 3 M_{\odot}$ from ZAMS mass $\sim 20 M_{\odot}$ in GRB060218/SN2006aj [86]. 


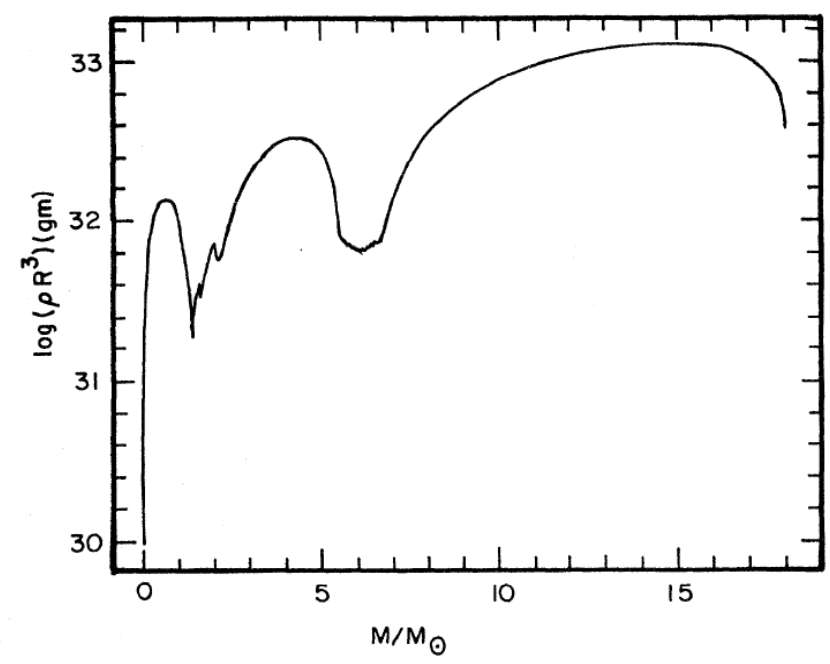

Fig. 6. Distribution of $\rho R^{3}$ vs included mass in the presupernova model of a star of mass $18 M_{\odot}$, according to Woosley [87]

\subsection{Accretion Onto Neutron Star After Supernova Explosion}

The shock wave powering the supernova explosion moves outwards through the helium core, essentially as a Sedov self-similar shock of constant velocity, the pressure being essentially inversely proportional to the volume. However $\rho R^{3}$, where $\rho$ is the density and $R$ is the radial distance from the center increase greatly in the hydrogen envelope (see Fig. 6). The shock has to slow down and the material behind it likewise [87].

The shock wave in the supernova after the first four seconds will be slowed down as it has to pick up more and more material in the mantle and envelope. The outgoing material in the shock wave thus has to be decelerated. Within this material, there is a density discontinuity where the He mantle adjoins the $\mathrm{H}$ envelope. In the deceleration, the lighter $\mathrm{H}$ gas has to push the heavier He, the classical situation for RT instability.

The deceleration takes place because the product $\rho r^{3}$ increases going out. The main increase, from $7 \times 10^{31}$ to $1.2 \times 10^{33}$, takes place continuously in the $\mathrm{H}$ envelope, from $m_{r}=7-11 M_{\odot}$ (see Fig. 6 ). In the Sedov theory, a shock wave continues at constant speed if $\rho r^{3}=$ const because its velocity is

$$
U \sim(p / \rho)^{1 / 2}
$$

and $p$ is inversely proportional to the enclosed volume. But if $\rho r^{3}$ increases, the shock has to slow down, and the material behind it likewise. This deceleration gradually sharpens into an ingoing shock. 
Woosley's calculation [88] shows that when the outgoing shock enters the hydrogen envelope, the deceleration of the shock sharpens into a reverse shock and this shock has been used to deposit $\sim 0.1 M_{\odot}$ back on the compact object [89]. Fig. 3 of Shigeyama et al. [90] shows the matter being deposited back on the neutron star.

When material sent radially inward by the reverse shock reaches the supernova center, the compact object is no longer there in case the neutron star receives a kick velocity. The material proceeds to move outwards from the original center. Eventually all of the matter will be moving radially outward, through with different speeds depending on the distance from the center. Thus Chevalier [91] suggests that the compact object will end up traveling at the same speed as its kick velocity. Therefore, we may, to a good approximation, consider the compact object to be at rest with respect to its ambient matter and use the Bondi [57] spherical accretion theory to determine $\dot{M}$, the rate of mass accretion onto the compact object. Brown and Weingartner [50] find that the photons from the initial accretion are trapped by the high density infalling matter, but that in $\sim 0.6$ yr one would have accretion at basically the Eddington limit.

\subsection{Observability Premium For Unseen Black Hole - Neutron Star Binaries}

Our prediction on the implication of kaon condensation on neutron stars in a binary has important ramifications on the number of light-mass black holes. We claim that there should be $\gtrsim 5$ times more low-mass black-hole, neutronstar binaries than double neutron-star binaries.

Now what is the status of the observation?

The observed pulsars have all been "recycled". That is, they have accreted mass from their companion. Empirically, this accretion of mass [92] brings down the magnetic field of the pulsar. The time that a pulsar can be seen is inversely proportional to its magnetic field. If the magnetic field is brought down a factor of $\sim 100$ from the recycling, the pulsar will be observable for 100 times longer. It is convenient to introduce the idea of "observability premium" [93]

$$
\Pi=\frac{10^{12} \mathrm{G}}{B}
$$

where $B$ is the magnetic field of the pulsar. $\Pi \simeq 1$ for fresh, unrecycled pulsars, which would appear in neutron star, black hole binaries, because the black hole cannot transfer mass ("recycle") the neutron star. For PSR 1913+16, the 
Hulse-Taylor binary, $B \sim 10^{10} \mathrm{G}$ for the pulsar, so it will be observable for $\sim 100$ times longer than the black hole, neutron star binary. In Table 1 we have 6 double neutron star binaries. Taking 100 to be a typical difference between the observable times for recycled and unrecycled pulsars, we see that we should see $\sim 0.6$ neutron-star, black-hole binaries. In fact, the factor is probably somewhat less than this because the observer would be somewhat biased against claiming something that cannot be directly seen, but identifiable only by its effects, such as a black hole in a binary.

An interesting recent development is the discovery of "twins" [94]. These authors show that "Binaries like to be twins"; i.e., something in their birth process seems to favor binaries in which the two stars are essentially identical. If this is true, it would enhance the double neutron star production, because it increases the number of neutron star pairs that are within $4 \%$ of each other in mass. They did not, however, correct for selection effects, which will probably lower somewhat their estimated number of twins.

Prompted by the paper on twins by Pinsonneault and Stanek [94], Lee et al. [66] made an improved calculation of the ratio of neutron star, low-mass black hole mergers to double neutron star mergers. The latter could be run up if there were lots of twins. However selection effects not included in [94] would have to be taken into account before one could estimate the number quantitatively. In the non-twin population, Lee et al. found that the number of low-mass black-hole, neutron star binaries would be $\sim 5$ times greater than double neutron-star binaries. For detection by LIGO the "chirp" mass would be somewhat greater for the former than the latter, because of the greater mass for the black holes.

In summary, it is clear to us that there are $\gtrsim 5$ times more low-mass black hole binaries than double neutron star binaries. As of now, the merging of these cannot be distinguished in the short-hard $\gamma$-ray bursts. However the consequences in terms of the two neutron stars in a binary having to be within $4 \%$ of each other in mass, with small correction made where there has been some He red giant mass transfer, are growing in number. We have explained why, in the case of SN1987A, the absence of evidence is evidence for a black hole.

\section{CONCLUSIONS}

We have been able to arrive at the strangeness condensation phase transition critical point $n_{c} \lesssim 3 n_{0}$ from three different starting points, the first from the

zero-density vacuum, the second from the vector manifestation fixed point and the third from the Landau Fermi-liquid fixed point. In the two last cases, 
the estimate was made more reliable by arguments based on renormalization group flow which made all of the quantities whose behavior with density was unknown rotated out. In particular in the second case, rotated out were the role of the scalar condensate $\langle\bar{q} q\rangle$, the explicit chiral symmetry breaking through $\Sigma_{K N}$, the role of strange baryons $\Lambda, \Sigma, \Xi$, the $\Lambda(1405)$ which was often viewed as the doorway state for kaon condensation, all of which figure importantly if one starts from the $T=n=0$ vacuum. We therefore favor the second scenario over the others. (In [20] a detailed discussion of these matters is given.)

We have shown that the hidden local symmetry in the "vector manifestation" (HLS-VM) prescribes the medium dependence imposed by (Wilsonian) matching to QCD of the quantities entering into the strangeness condensation. In particular, since HLS-VM is a fixed point with vanishing gauge coupling constant, chiral perturbation theory is well-defined. Assuming that HLS theory represents QCD near the fixed point, one can efficiently calculate the strangeness condensation by fluctuating about the fixed point. It turns out that the only degree of freedom we need to take into account is the vector degree of freedom in the form of on-shell constituent quarks. In other words, the strangeness condensation can be calculated in the constituent quark model, with the strange quark a passive observer.

Although we are at a very early stage of understanding, the recent development of holographic QCD, in particular, the Sakai-Sugimoto model, that presumably encapsulates QCD at low energy in terms of an infinite tower of hidden local fields promises to confirm or invalidate our main theme. Integrating out the higher members of the tower leaving the lowest could lead to HLS-VM theory of Harada and Yamawaki, with the vector manifestation representing the effect of higher members of the tower when constrained to QCD. At present, it is not known how to go beyond the large $N_{c}$ and large 't Hooft limit in the Sakai-Sugimoto model but once one knows how to handle certain corrections in the dual sector with appropriate matching to gauge theory QCD, the characteristic feature of the vector manifestation such as the hadronic freedom on which our argument relies could be verified.

In a neutron star the $K^{-}$chemical potential $\mu_{K^{-}}$, essentially the $K^{-}$mass, will be lowered by the attractive interaction between the quarks in the neutrons and protons, chiefly by the former, so that at a certain density, which we estimate to be $3 n_{0}$, the $\mu_{K^{-}}$becomes equal to $\mu_{e^{-}}$. At that point the electrons change into $K^{-}$mesons, which go into an S-wave Bose condensate. With the accompanying lowering of pressure, the star drops into a black hole. This we believe is the first phase transition dense compact star matter undergoes which determines the fate of star, that is whether it becomes a neutron star or a black hole.

We believe that this scenario describes the fate of the supernova explosion 
SN1987A. What we are sure of is that in the evolution of binary neutron stars, the first-born neutron star will go into a black hole if the two progenitor giants are not within $4 \%$ of each other in mass. (For the low-mass neutron-star binary the situation is slightly shifted, as we explained in the text.)

Since our main early interest in kaon condensation was in the disappearance of evidences of the existence of neutron star in SN1987A after it had emitted neutrinos for about 12 seconds, we felt it fitting to adduce even more firm astrophysical observations that shows that there are a large number of lowmass black holes in the Universe. Most specific is that we showed that double neutron stars in binaries must be within $4 \%$ of each other in mass; otherwise the binary would end up being one of a neutron star and a low-mass black hole. Observations support the necessity of the neutron star being within $4 \%$ of each other in mass within any given binary. This also means that there are $\sim 5$ times more neutron-star, low-mass black hole binaries than double neutron-star binaries in the Galaxy.

Finally, putting together all of the above leads us back to the proposal of Brown and Bethe [37] that the maximum neutron star mass is $\sim 1.5 M_{\odot}$, not much higher than the mass of the Hulse-Taylor pulsar $1913+17$ of $1.44 M_{\odot}$. This is the only accurately measured mass of the more massive neutron stars. We believe, for example, the probability that J0751+1807, of mass $2.1 \pm 0.2$ but with $95 \%$ probability $>1.6 M_{\odot}$ has mass $\sim 1.5 M_{\odot}$ to be substantially higher than for the spectrum of neutron stars in binaries that would exist were $2.1 M_{\odot}$ neutron stars to be stable. The announced reduction of the measured mass of $\mathrm{J} 0751+1807$ to $\sim 1.26 M_{\odot}$ goes in the direction consistent with the above reasoning.

The Harada and Yamawaki HLS-VM had predictions that countered well established beliefs. Perhaps the most controversial was the prediction in HLSVM of hadronic freedom, that the interactions between hadrons go to zero as the temperature goes up to $T_{\chi}$ from below and the density goes up to $n_{\chi}$ from $n_{0}$. This directly contradicted the accepted belief in heavy-ion community that interactions between hadrons became more plentiful and stronger as $T$ goes up to $T_{c}$ from below. We managed to find what we believe to be the crucial experiment to decide this issue, namely the STAR experiment of peripheral $\mathrm{Au}+\mathrm{Au}$ collisions which measured $\rho^{0} / \pi^{-}$ratio [26]. We discussed this experiment briefly in Sec. 5. The experiment was so useful because in this peripheral experiment the freeze out temperature was equal to the flash temperature, the temperature at which the hadrons went on shell. The results of this STAR experiment directly supported the concept of hadronic freedom in temperature. We are proposing that the same is the case in density and applies to kaon condensation. We found in this paper that HLS-VM gave unambiguous statements about how to handle the medium dependencies and that is why we are so sure of the results. Needless to say, the crucial element 
in this argument is the existence of the vector manifestation (VM) fixed point which requires the vanishing of the vector meson masses (in the chiral limit). The VM in Harada-Yamawaki HLS theory is an unambiguously falsifiable prediction: it could be invalidated if an unquenched lattice measurement of the spectral function of the $\rho$ channel unquestionably showed that the (real-time) mass of the $\rho$ meson does not vanish in the chiral limit at $T=T_{\chi}$.

The question we have not addressed here is this: if kaon condensation is inevitable in compact stars once density reaches $\sim 3 n_{0}$, will other forms of exotic states that can take place at higher densities, say, $n>n_{c} \sim 3 n_{0}$, such as color superconductivity, color flavor locking etc. be relevant for the physics of compact stars? We can offer no definitive answer to this question. Our conjecture is that they will be hidden in the "black-hole nothingness" inaccessible to the observers. The question can perhaps be answered when holographic QCD becomes sufficiently sophisticated and predictive.

\section{Acknowledgments}

G.E.B. was supported in part by the US Department of Energy under Grant No. DE-FG02-88ER40388. C.H.L. was supported by Creative Research Initiatives (MEMS Space Telescope) of MOST/KOSEF.

\section{References}

[1] M. Harada and K. Yamawaki, Phys. Rept. 381 (2003) 1.

[2] G.E. Brown and M. Rho, Phys. Rev. Lett. 66 (1991) 2720.

[3] S. Weinberg, Physica A96 (1979)327; "What is quantum field theory, and what did we think it is?," hep-th/9702027.

[4] See, e.g., M. Rho, Chiral Nuclear Dynamics II: From Quarks to Nuclei to Compact-Star Matter, World Scientific, to appear.

[5] D.B. Kaplam and A.E. Nelson, Phys. Lett. B175 (1986) 57.

[6] G.E. Brown, K. Kubodera and M. Rho, Phys. Lett. B192 (1987) 273.

[7] J.J. Dong, J.-F. Lagaë and K.F. Liu, Phys. Rev. D54 (1996) 5496.

[8] C.-H. Lee, Phys. Rept. 275 (1996) 255.

[9] T. Waas and W. Weise, Nucl. Phys. A625 (1997) 287.

[10] V. Thorsson, M. Prakash, and J.M. Lattimer, Nucl. Phys. A 572 (1994) 693. 
[11] G.E. Brown, V. Thorsson, K. Kubodera and M. Rho, Phys. Lett. B291 (1992) 355.

[12] H.K. Lee, M. Rho and S.-J. Sin, Phys. Lett. B348 (1995) 290.

[13] R. Shankar, Rev. Mod. Phys. 66 (1994) 129.

[14] J. Carlson, H. Heiselberg and V.R. Pandharipande, Phys. Rev. C63 (2001) 017603.

[15] M. Harada and C. Sasaki, Phys. Lett. B537 (2002) 280.

[16] M. Harada, Y. Kim and M. Rho, Phys. Rev. D 66 (2002) 016003.

[17] M. Harada, M. Rho and C. Sasaki, Phys. Rev. D70 (2004) 074002.

[18] M. Harada, M. Tanabashi and K. Yamawaki, Phys. Lett. B568 (2003)103.

[19] G.E. Brown and M. Rho, Phys. Repts. 396 (2004) 1.

[20] G.E. Brown, C.-H. Lee, H.-J. Park and M. Rho, Phys. Rev. Lett. 96 (2006) 062303.

[21] T. Sakai and S. Sugimoto, Prog. Theor. Phys. 113 (2005) 843; 114 (2005) 1083.

[22] D.K. Hong, M. Rho, H.-Y. Yee and Y. Yi, JHEP 09 (2007) 063.

[23] H.-J. Lee, B.-Y. Park, D.-P. Min, M. Rho and V. Vento, Nucl.Phys. A723 (2003) 427; H.-J. Lee, B.-Y. Park, M. Rho and V. Vento, Nucl.Phys. A726 (2003) 69, H.-J. Lee, B.-Y. Park, M. Rho and V. Vento, Nucl.Phys.A741 (2004) 161.

[24] R. Alkofer, S.T. Hong and I. Zahed, J. Phys. G: Nucl. Part. 17 (1991) L59.

[25] G.E. Brown, C.-H. Lee, and M. Rho, Phys. Rev. C 74 (2006) 024906.

[26] J. Adams et al. (STAR Collaboration), Phys. Rev. Lett. 92 (2004) 092301.

[27] M. Harada and C. Sasaki, Nucl. Phys. A736 (2004) 300

[28] M. Rho, "Hidden local symmetry and dense half-skyrmion matter," e-Print: arXiv:0711.3895 [nucl-th]

[29] A. Akmal, V.R. Pandharipande and D.G. Ravenhall, Phys. Rev. C58 (1998) 1804.

[30] G.E. Brown, J.W. Holt, C.-H. Lee and M. Rho, Phys. Rept. 439 (2007) 439.

[31] K. Suzuki et al., Phys. Rev. Lett. 92 (2004) 072302.

[32] E. Friedman and A. Gal, "In medium nuclear interactions of low-energy hadrons", Physics Reports, in press.

[33] M. Harada and C. Sasaki, Phys. Rev. D74 (2006) 114006.

[34] M. Prakash, J.R. Cooke, J.M. Lattimer, Phys. Rev. D52 (1995) 661.

[35] G.E. Brown, C.-H. Lee, and R. Rapp, Nucl. Phys. A 639 (1998) 455c. 
[36] N.K. Glendenning, Phys. Rep. 342 (2001) 393.

[37] G.E. Brown and H.A. Bethe, ApJ, 423 (1994) 659.

[38] G.E. Brown, Astrophys. J. 440 (1995) 270.

[39] R.A. Chevalier, Astrophys. J. Letters 411 (1993) 33.

[40] B. Willems and V. Kalogera, ApJ. 603 (2004) L101.

[41] J.M. Lattimer and M. Prakash, Astrophys. J. 550 (2001) 426;

[42] F. Weber, Prog. Part. Nucl. Phys. 54 (2005) 193;

[43] J.M. Lattimer and M. Prakash, Nucl. Phys. A777 (2006) 479;

[44] H.Th. Janka, K. Kifonidis, M. Rampp, Lect. Notes in Phys. 578 (2001) 333;

[45] A. Burrows, L. Dessart, C.D. Ott, Eli Livne, Phys. Reports 442 (2007) 23;

[46] H.Th. Janka et al. Phys. Reports 442 (2007) 38;

[47] A. Mezzacappa, Ann. Rev. Nucl. Part. Sci. 55 (2005) 467;

[48] K. Postnov and L. Yungelson, Living Reviews in Relativity, Lrr-2006-6.

[49] J.M. Blondin, ApJ. 308 (1986) 755.

[50] G.E. Brown and J.C. Weingartner, ApJ 436 (1994) 843.

[51] J.C. Houck and R.A. Chevalier, ApJ 376 (1991) 234.

[52] G.E. Brown, C.-H. Lee and H.A. Bethe, ApJ 541 (2000) 918.

[53] K. Belczynski, V. Kalogera, and T. Bulik, ApJ 572 (2002) 407.

[54] Ya. B. Zel'Dovich, L.N. Ivanova, and D.K. Nadezhin, Soviet Astronomy 16 (1972) 209.

[55] P.J. Armitage and L. Livio, ApJ. 532 (2000) 540.

[56] H.A. Bethe and G.E. Brown, ApJ. 506 (1998) 780.

[57] H. Bondi, Mon. Not. Roy. Astron. Soc. 112 (1952) 755.

[58] J.L. Terman, R.E. Taam, L. Hernquist, ApJ 422 (1994) 729.

[59] H. Braun and N. Langer, A\&A 297 (1995) 483.

[60] J. Lattimer and M. Prakash, Physics Reports 442 (2007) 166.

[61] S.E. Thorsett and D. Chakrabarty, ApJ 512 (1999) 288.

[62] A.G. Lyne, et al., Science 303 (2004) 1153.

[63] A.J. Faulkner et al. ApJL 618 (2004) L119. 
[64] J.D.M. Dewi and E.P.J. van den Heuvel, Mon.Not.Roy.Astron.Soc. 349 (2004) 169.

[65] B. Willems and V. Kalogera, ApJ 603 (2004) L101.

[66] C.-H. Lee, H.-J. Park, and G.E. Brown, ApJ, accepted; astro-ph/0607442.

[67] J. Lattimer and M. Prakash, Science 304 (2004) 536.

[68] H.A. Bethe and G.E. Brown, ApJ 445 (1995) L129.

[69] D.J. Nice et al., ApJ 634 (2005) 1242.

[70] F.K. Thielemann, K. Nomoto, and M. Hashimoto, "Origin and evolution of the elements: proceedings of a symposium in honour of $\mathrm{H}$. Reeves, held in Paris, June 22-25, 1992. Edited by N. Prantzos, E. Vangioni-Flam and M. Casse (Cambridge University Press, 1993), p. 297.

[71] F.X. Timmes, S.E. Woosley, T.A. Weaver, ApJ 98 (1995) 617.

[72] T.M. Tauris and G.J. Savonije, A\&A 350 (1999) 928.

[73] T.M. Tauris, E.P.J. van den Heuvel, and G.J. Savonije, ApJ 530 (2000) L93.

[74] S.M. Ransom, et al., Science 307 (2005) 892.

[75] P. Haensel, M. Bejger, and J.L. Zdunik, asXiv:0705.4594 (astro-ph).

[76] H.A. Bethe, G.E. Brown and C.-H. Lee, Phys. Repts. 442 (2007) 5.

[77] R. Kunz, M. Jaeger, A. Mayer, J.W. Hammer, G. Staudt, S. Harissopulos, and T. Paradellis, Phys. Rev. Lett. 86 (2001) 3244.

[78] L.R. Buchmann and C.A. Barnes, Nucl. Phys. A 777 (2006) 254.

[79] H.A. Bethe, G.E. Brown, J. Applegate and J.M. Lattimer, Nuclear Physics A324 (1994) 487.

[80] G.E. Brown, A. Heger, N. Langer, C.-H. Lee, S. Wellstein, and H.A. Bethe, New Astronomy 6 (2001) 457.

[81] A. Heger, S.E. Woosley, G. Martinez-Pinedo, and K. Langanke, ApJ 560 (2001) 307.

[82] S.E. Woosley and T.A. Weaver, ApJ 101 (1995) 181.

[83] K. Langanke and G. Martinez-Pinedo, Nucl. Phys. A 673 (2000) 481.

[84] S.E. Thorsett and D. Chakrabarty, ApJ 512 (1999) 288.

[85] C.-H. Lee, G.E. Brown, and R.A.M.J. Wijers, ApJ 575 (2002) 996.

[86] G.E. Brown, C.-H. Lee, and R.A.M.J. Wijers, in preparation.

[87] H.A. Bethe, Rev. Mod. Phys. 62 (1990) 801.

[88] S.E. Woosley, ApJ 330 (1988) 218. 
[89] R.A. Chevalier, ApJ 246 (1981) 847.

[90] T. Shigeyama, K. Nomoto, and M. Hashimoto, A\&A 196 (1988) 141.

[91] R.A. Chevalier, ApJ, 346 (1989) 847.

[92] R.E. Taam and E.P.J. van den Heuvel, ApJ 350 (1986) 235.

[93] T. Wettig and G.E. Brown, New Astronomy 1 (1996) 17.

[94] M.H. Pinsonneault and K.Z. Stanek, ApJ 639 (2006) L67. 